\title{
Equation of State and Glass Transition of Stretched Network Polymers
}

\author{
Takuhei NosE \\ Department of Polymer Chemistry, Tokyo Institute of Technology, \\ Ookayama, Meguro-ku, Tokyo, Japan.
}

(Received March 4, 1972)

\begin{abstract}
The glassy state and the glass transition of a stretched network polymers are studied on the basis of the hole theory introducing the concept of ordering parameters in non-equilibrium thermodynamics, where the hole and the conformation of network chain are supposed to be frozen-in in the glassy state. The equations for the change in glass transition temperature with stretching are derived to test the criteria for glass transition, i.e., the iso-free volume, the iso-configurational entropy, and the iso-configurational energy assumptions. It was found that the second assumption explains the experimental facts best. The enthalpy and volume of the glass having histories of stretching are also evaluated. The difference in the enthalpy between stretched and non-stretched glasses depends not only on the change in the conformational energy with stretching but also on the differences in volume and glass-forming temperature.

KEY WORDS Polymer / Glassy State / Equation of State / Glass

Transition / Stretch / Enthalpy /
\end{abstract}

In order to understand the glass transition and glassy state of amorphous polymers, the author has already investigated, on the basis of the hole theory, the pressure effects on the glass transition, and the thermodynamic behaviors of those glasses of different thermal and pressure histories. ${ }^{1}$ It was concluded that the glass transition point is not the iso-free volume state, but near the isoconfigurational entropy or energy state, and it was suggested that the chain flexibility also plays a role in the segmental motion as important as the free volume. In this paper, stretched network polymers are studied using the same treatment as in a previous paper. ${ }^{1}$ The present study has two main aims; one is to derive the thermodynamic relations for the change in the glass transition temperature with stretching in order to test the criteria for the glass transition, and the other is to derive the expressions for the thermodynamic quantities of the glass having histories of stretching.

The treatment of the glass transition temperature and the glassy state adopted here is the same as that used in a previous paper, ${ }^{1}$ and is described in general terms as follows.

First one defines the two kinds of parameters which determine the thermodynamic state of a system specified, for example, by the free energy
$F$; one is not frozen and the other is frozen in the glassy state. Let us denote the former by $x_{1}, x_{2}, \ldots, x_{i}, \ldots, x_{n}$, and the latter by $y_{1}$, $y_{2}, \ldots, y_{i}, \ldots, y_{m}$. All of the former quantities are not generally independent. When the temperature $T$ and volume $V$ are taken as thermodynamic variables, $x_{i}$ is supposed to be determined by giving the set of variables $\left(T, V, y_{1}, \ldots, y_{m}\right)$. The latter one, $y_{i}$, corresponds to the ordering parameters or internal variables in the thermodynamic treatments of relaxation phenomena presented by Meixner and Davies-Jones et al. ${ }^{2}$

The total differential of Helmholtz free energy $F$ is then written as

$$
\begin{aligned}
\mathrm{d} F= & \sum_{i=1}^{n}\left(\partial F / \partial x_{i}\right)_{x_{j \neq i}, y} \mathrm{~d} x_{i}+\sum_{i=1}^{m}\left(\partial F / \partial y_{i}\right)_{x, y_{j \neq i}} \mathrm{~d} y_{i} \\
= & \sum_{i=1}^{n}\left(\partial F / \partial x_{i}\right)_{x_{j \neq i}, y}\left\{\left(\partial x_{i} / \partial T\right)_{V, y} \mathrm{~d} T\right. \\
& \left.+\left(\partial x_{i} / \partial V\right)_{T, y} \mathrm{~d} V\right\}-\sum_{i=1}^{m} A_{i} \mathrm{~d} y_{i}
\end{aligned}
$$

where $A_{i}$ is the affinity for $y_{i}$ defined by

$$
\begin{aligned}
A_{i} \equiv & -\left(\partial F / \partial y_{i}\right)_{T, V, y_{j \neq i}}=-\left\{\left(\partial F / \partial y_{i}\right)_{x, y_{j \neq i}}\right. \\
& \left.+\sum_{k=1}^{n}\left(\partial F / \partial x_{k}\right)_{x_{j \neq i}, y}\left(\partial x_{k} / \partial y_{i}\right)_{T, V, y_{j \neq i}}\right\} .
\end{aligned}
$$


In the liquid state, which is an equilibrium state, the affinity is zero;

$$
A_{i}=0 \text {. }
$$

On the other hand, in the glassy state,

$$
y_{i}=y_{i g}=\text { constant }
$$

or

$$
\mathrm{d} y_{i}=0
$$

where $y_{i \mathrm{~g}}$ is $y_{i}$ at which the system is frozen-in, i.e., $y_{i}$ at the glassforming point.

On the other hand, since the system is specified by the set of parameters $\left(x_{1}, \ldots, x_{n}, y_{1}, \ldots, y_{m}\right)$, the total differential of a thermodynamic quantity $\boldsymbol{X}$ is also expressed in general as

$\mathrm{d} X=\sum_{i=1}^{n}\left(\partial X / \partial x_{i}\right)_{x_{j \neq i}, y} \mathrm{~d} x_{i}+\sum_{i=1}^{m}\left(\partial X / \partial y_{i}\right)_{x, y_{j \neq i}} \mathrm{~d} y_{i}$.

The conditions of eq 3 and 4 (or 5) lead to the thermodynamic quantities for the liquid and glassy states. For example, the equation of state for an unstretched substance is given from eq 1 or $1^{\prime}$ by

$$
P=-(\partial F / \partial V)_{T}=-\sum_{i=1}^{n}\left(\partial F / \partial x_{i}\right)_{x_{j \neq i}, y}\left(\partial x_{i} / \partial V\right)_{T, y}
$$

with $A_{i}=0$, for liquid state, and

$$
\boldsymbol{P}=-\sum\left(\partial F / \partial x_{i}\right)_{x_{j \neq i}, y=y_{\mathrm{g}}}\left(\partial x_{i} / \partial V\right)_{T, y=y_{\mathrm{g}}}
$$

with $y_{i}=y_{i g}$, for the glassy state, where $P$ is pressure. The difference in the change of thermodynamic quantity $X$ between the liquid and glassy states is expressed from eq 6 with eq 3 and 5 as

$$
\begin{aligned}
\Delta(\mathrm{d} X) \equiv & (\mathrm{d} X)^{1}-(\mathrm{d} X)^{\mathrm{g}}=(\mathrm{d} X)_{A=0}-(\mathrm{d} X)_{y} \\
= & \sum_{i=1}^{n}\left(\partial X / \partial x_{i}\right)_{x_{j \neq i}, y} \Delta\left(\mathrm{d} x_{i}\right) \\
& +\sum_{i=1}^{m}\left(\partial X / \partial y_{i}\right)_{x, y} \ldots i=1
\end{aligned}
$$

Here, the symbol $\Delta$ designates the difference between the liquid and glassy states, the superscripts 1 and $g$ represent the quantities in the liquid and glassy states, respectively, and the subscripts $A=0$ and $y$ indicate the fixed variables in the differentials.

In the previous treatments, another assumption was introduced in order to define the configurational part of a thermodynamic quantity, entropy or internal energy, which is frozen in the glassy state, as a function of state. Namely, the free energy of a system was assumed to be formally expressed as

$$
\begin{aligned}
F= & U^{\mathrm{c}}\left(y_{1}, y_{2}, \ldots, y_{m}\right)-T S^{\mathrm{c}}\left(y_{1}, y_{2}, \ldots, y_{m}\right) \\
& +U^{*}\left(x_{1}, x_{2}, \ldots, x_{n}\right)-T S^{*}\left(x_{1}, x_{2}, \ldots, x_{n}\right)
\end{aligned}
$$

where $U^{\mathrm{c}}$ is the configurational energy, and $S^{\mathrm{c}}$ the configurational entropy. This assumption for the form of $F$ was required in order to define $S^{\mathrm{c}}$ (or $U^{c}$ ) as a part of $S($ or $U$ ) which is frozen-in in the glassy state and is determined by $y$ only. The frozen parameters $y$ specify the configuration of chain molecules in the system. Unless the part of the entropy related to the configuration can be separately evaluated (as in eq 10) at least approximately, "the configurational entropy" will loss its meaning. It was deduced from this assumption, as indicated previously in the hole model, ${ }^{1}$ that the thermal expansion coefficient $\alpha$, compressibility $\beta$ and heat capacity $C_{p}$ of a glass are almost independent of the frozen parameters which are determined by the condition of the glass formation. If there exists a term of $S$ (and/ or $U$ ) which depends strongly both on $x$ and $y$ and can not be expressed separately as the form of eq 10 , such quantities as $\alpha, \beta$ and $C_{p}$ of a glass should depend strongly on $y$, i.e., on the history of the glass. The assumption of eq 10, therefore, also implies that $\alpha, \beta$, and $C_{p}$ are almost independent of the history of the glass, in accordance with experiments. This is also supported by the fact that the excess entropy of the glassy state relative to the crystalline state is almost independent of temperature. Since the crystalline state

* When $X$ is regarded as a function of $\left(T, P, y_{1}\right.$, $\left.\ldots, y_{m}\right)$, one has an alternative form equivalent to. eq 9 using eq 3,5 , and 6 ;

$$
\Delta(\mathrm{d} X)=\sum_{i=1}^{m}\left(\partial X / \partial y_{i}\right)_{T, P}\left(\mathrm{~d} y_{i}\right)_{A=0}
$$

which is reformed as

$$
\begin{aligned}
\Delta(\mathrm{d} X)= & \sum_{i=1}^{m}\left\{\sum_{k=1}^{n}\left(\partial X / \partial x_{k}\right)_{x}, k_{j \neq},\left(\partial x_{k} / \partial y_{i}\right)_{T, P}\right. \\
& \left.+\left(\partial X / \partial y_{i}\right)_{x, y_{j \neq i}}\right\}\left(\mathrm{d} y_{i}\right)_{A=0} \\
= & \sum_{k=1}^{n}\left(\partial X / \partial x_{k}\right)_{j \neq k}, y \sum_{i=1}^{m}\left(\partial x_{k} / \partial y_{i}\right)_{T, P}\left(\mathrm{~d} y_{i}\right)_{A=0} \\
& +\sum_{i=1}^{m}\left(\partial X / \partial y_{i}\right)_{x, y_{j \neq i}}\left(\mathrm{~d} y_{i}\right)_{A=0} \\
= & \text { the right hand side of eq } 9
\end{aligned}
$$


Equation of State and Glass Transition of Stretched Network Polymers

can be regarded as a kind of frozen state. The conventional way to evaluate the configurational entropy $S^{\mathrm{c}}$, i.e., the estimation of $S^{\mathrm{c}}$ by the equation

$$
S^{\mathrm{c}}(T)=S^{\mathrm{c}}\left(T_{0}\right)+\int_{T_{0}}^{T}\left(\Delta C_{p} / T\right) \mathrm{d} T,
$$

where $\Delta C_{p}$ is the difference in $C_{p}$ between the liquid and crystalline states, is essentially based on the assumption of eq 10 with $\Delta\left(\mathrm{d} x_{i} / \mathrm{d} T\right)=0$ as seen in eq 12 described below. Under the assumption of eq $10,\left(\mathrm{~d} Z^{\mathrm{c}}\right)^{1}(Z=S$ or $U)$ can be written as

$$
\left(\mathrm{d} Z^{\mathrm{c}}\right)^{1}=\sum_{i=1}^{m}\left(\partial Z / \partial y_{i}\right)_{x, y_{j \neq i}}\left(\mathrm{~d} y_{i}\right)_{A=0}
$$

Therefore, from eq 9 the change in the configurational quantity $\left(\mathrm{d} Z^{\mathrm{c}}\right)^{1}$ is expressed as

$$
\left(\mathrm{d} Z^{\mathrm{c}}\right)^{1}=\Delta(\mathrm{d} Z)-\sum_{i=1}^{n}\left(\partial Z / \partial x_{i}\right)_{x_{j \neq i}, y} \Delta\left(\mathrm{d} x_{i}\right)
$$

Generally speaking, $\Delta\left(\mathrm{d} x_{i}\right)$ is not zero, and it must be noticed therefore that the change in the configurational part of the thermodynamic quantity is not always expressed as the difference in the change in the thermodynamic quantity between the liquid and glassy states.

The change of $X_{s}$ with $Y_{t}$ at the glass transition point, $\left(\partial Y_{s} / \partial Y_{t}\right)_{\mathrm{g}}$, is expressed in terms of $(\partial X /$ $\left.\partial Y_{t}\right)_{\mathrm{g}}$, or $\left(\partial X / \partial Y_{s}\right)_{\mathrm{g}}$, as

$$
\begin{aligned}
& \left(\partial Y_{s} / \partial Y_{t}\right)_{\mathrm{g}} \\
& =\left\{-\left(\partial X / \partial Y_{t}\right)_{Y_{i \neq t}}+\left(\partial X / \partial Y_{t}\right)_{\mathrm{g}}\right\} /\left(\partial X / \partial Y_{s}\right)_{Y_{i \neq s}} \\
& =-\left(\partial X / \partial Y_{t}\right)_{Y_{i \neq t}} /\left\{\left(\partial X / \partial Y_{s}\right)_{Y_{i \neq s}}-\left(\partial X / \partial Y_{s}\right)_{\mathrm{g}}\right\}
\end{aligned}
$$

when the thermodynamical variables other than $Y_{s}$ and $Y_{t}$ are fixed.

In the preceding paper, ${ }^{1}$ to deal with the change of the glass transition temperature with pressure, a hole model was adopted and the variables chosen as

$$
x_{i}: V^{*}, T, \quad y_{i}: \tilde{V}, S_{1}{ }^{\mathrm{c}}, \quad Y: Y_{s}=T, Y_{t}=P
$$

where $V^{*}$ is the volume of the cells occupied with segments, $\tilde{V}$ is defined as $V / V^{*}$, and $S_{1}{ }^{\mathrm{c}}$ is the conformational entropy of polymer chains. Since it is derived from eq 10 that $\left(\partial V^{*} / \partial T\right)_{p}$ and $\left(\partial V^{*} /\right.$ $\partial P)_{T}$ are continuous at the glass transition temperature, ${ }^{1}$ it follows from eq 12 with $Z=S$ that

$$
\left(\partial S^{\mathrm{c}} / \partial T\right)_{p}{ }^{1}=\Delta(\partial S / \partial T)_{p}=\Delta C_{p} / T
$$

and

$$
\left(\partial S^{\mathrm{c}} / \partial P\right)_{T}{ }^{1}=\Delta(\partial S / \partial P)_{T}=-V \Delta \alpha
$$

where $C_{p}$ is heat capacity under constant pressure $T(\partial S / \partial T)_{P}$, and $\alpha$ thermal expansion coefficient $(\partial \ln V / \partial T)_{p}$. Then, substituting eq 14 and 15 into eq 13 with $X=S^{\mathrm{c}}$, one has

$$
(\partial T / \partial P)_{\mathrm{g}}=\left\{T V \Delta \alpha+T\left(\partial S^{\mathrm{c}} / \partial P\right)_{\mathrm{g}}\right\} / \Delta C_{p} .
$$

This is one of the equations for $(\partial T / \partial P)_{\mathrm{g}}$ obtained in a previous paper. ${ }^{1}$ If the iso- $S^{\mathrm{c}}$ at the glass transition point is a valid assumption, i.e., $\left(\partial S^{\mathrm{c}} / \partial P\right)_{\mathrm{g}}=0$, the relation $(\partial T / \partial P)_{\mathrm{g}}=T V \Delta \alpha / \Delta C_{p}$ is derived from eq 16.

\section{THEORETICAL}

\section{Model}

A rectangular network polymer, consisting of $N$ segments, of volume $V=\prod_{i=1}^{3} L_{i}$ is considered, where $L_{i}$ is the length in the $i$-th direction, and 1-direction $(i=1)$ is taken as the direction of stretching. In the system, where $M$ lattice sites are involved, there remain $(M-N)$ empty sites or holes. If the cell volume is denoted by $v^{*}$, $V \equiv M v^{*}$, and $V^{*}$ is defined as $V^{*} \equiv N v^{*}$. Then, $\tilde{V} \equiv V / V^{*}=M / N$, and $\left(1-\tilde{V}^{-1}\right)$ represents the hole fraction. The cell dimensions are not necessarily isotropic and can be assumed to be expressed by $v^{*}=\prod_{i=1}^{3} l_{i}{ }^{*}$, where $l_{i}{ }^{*}$ is the representative length in the $i$-th direction of the cell. The quantity $m_{i}$ is then defined as $L_{i}=m_{i} l_{i}{ }^{*}$, and thus $M=$ $\prod_{i=1}^{3} m_{i}$. The occupied volume $V_{0}=N v_{0}$ is assumed to be a constant independent of temperature, external pressure, and deformation. As the quantity which expresses the expansion of the cell volume, $V^{+}$is defined by $V^{+} \equiv V^{*} / V_{0}=\prod_{i=1}^{3}\left(l_{i}{ }^{*} /\right.$ $\left.v_{0}{ }^{1 / 3}\right)=\prod_{i=3}^{3} l_{i}{ }^{+}$. Summing up the relations among the quantities defined above;

$$
\begin{aligned}
& V=\prod_{i=1}^{3} L_{i} \\
& V^{*}=N v^{*}=N \prod_{i=1}^{3} l_{i}^{*} \\
& V^{+}=V^{*} / V_{0}=v^{*} / v_{0}=\prod_{i=1}^{3} l_{i}^{+} \\
& l_{i}^{+}=l_{i}^{*} / v_{0}{ }^{1 / 3}
\end{aligned}
$$




$$
\begin{aligned}
& \tilde{V}=V / V^{*}=M / N=\prod_{i=1}^{3} m_{i} / N \\
& L_{i}=m_{i} l_{i}^{*}
\end{aligned}
$$

The total differential forms are;

$$
\begin{aligned}
\mathrm{d} \ln V & =\sum_{i=1}^{3} \mathrm{~d} \ln m_{i}+\sum_{i=1}^{3} \mathrm{~d} \ln l_{i}^{*} \\
& =\mathrm{d} \ln \tilde{V}+\sum_{i=1}^{3} \mathrm{~d} \ln l_{i}^{+} \\
\mathrm{d} \ln L_{i} & =\mathrm{d} \ln m_{i}+\mathrm{d} \ln l_{i}^{*} \\
\mathrm{~d} \ln \tilde{V} & =\sum_{i=1}^{3} \mathrm{~d} \ln m_{i} \\
\mathrm{~d} \ln l_{i}^{+} & =\mathrm{d} \ln l_{i}^{*} \\
\mathrm{~d} \ln V^{+} & =\sum_{i=1}^{3} \mathrm{~d} \ln l_{i}^{+}=\sum_{i=1}^{3} \mathrm{~d} \ln l_{i}^{*}
\end{aligned}
$$

In general, a distribution function for mean end-to-end vectors of network chains must be defined in order to specify the state of the chain conformations. The affine deformation is, however, assumed here, i.e., it is assumed that the mean crosslinking point-to-crosslinking point vector $\varepsilon$ for any chain is expressed in term of the displacement tensor $\boldsymbol{\gamma}$ as

$$
\begin{aligned}
& \varepsilon=\boldsymbol{\gamma} \cdot \varepsilon^{*}=\boldsymbol{r}^{\prime} \cdot \varepsilon_{0}=b a \cdot \lambda \cdot \varepsilon_{00} \\
& \boldsymbol{\gamma}^{\prime}=\boldsymbol{a} \cdot \lambda \\
& \varepsilon_{0}=b \cdot \varepsilon_{00}
\end{aligned}
$$

where $\varepsilon^{*}$ is $\varepsilon$ in the unstretched state, $\varepsilon_{0}$ is $\varepsilon$ when the chain is an uncrosslinked free chain at the same temperature and $\varepsilon_{00}$ is $\varepsilon_{0}$ at the temperature of crosslinking. $\boldsymbol{a}$ is associated with the change in $\varepsilon$ arising from variations of bond length and small deviations of rotational angles from stable positions (e.g., trans and gauche), being variable both in the glassy and liquid states, and $\lambda$ and $b$ are associated with the change in $\varepsilon$ caused by the variation of trans-gauche conformations, which are frozen-in in the glassy state. The former, $\lambda$, is related to stretching and the latter scalar quantity $b$ is a function of temperature alone, representing the change in the unperturbed chain dimension with temperature. In the present paper, only simple elongation is considered. Then, $\boldsymbol{a}$ and $\lambda$ are written as

$$
\boldsymbol{a}=\left(\begin{array}{lll}
a_{1} & 0 & 0 \\
0 & a_{2} & 0 \\
0 & 0 & a_{3}
\end{array}\right), \quad \lambda=\left(\begin{array}{lll}
\lambda_{1} & 0 & 0 \\
0 & \lambda_{2} & 0 \\
0 & 0 & \lambda_{3}
\end{array}\right)
$$

Therefore, from eq 28 and 31 , one has

$$
\mathrm{d} \ln L_{i}=\mathrm{d} \ln \lambda_{i}+\mathrm{d} \ln a_{i}+\mathrm{d} \ln b
$$

The glassy state is dealt with here as a quasiequilibrium state, i.e., it is regarded as a frozen liquid such that polymer chains cannot change their trans-gauche conformations and cannot exchange their mean positions with each other. Thus, in the glassy state, the lattice number and arrangement specified by $m_{i}$, accordingly the hole fraction specified by $\tilde{V}$ (eq 21), and the state of chain conformations specified by $\lambda$ and $b$ are assumed to be completely frozen-in. Namely, the quantities $m_{i}, \tilde{V}, \lambda$, and $b$ are constant in the glassy state, and correspond to in internal variables $y$ described in the introduction. Since $m_{1}$, $m_{2}, m_{3}$, and $\tilde{V}$ are related with each other by eq 25 , three of them are independent variables in general and can be chosen as $y$. On the other hand, $a_{i}$ and $l_{i}^{+}$are variable both in the liquid and glassy states, and correspond to the nonfrozen parameters $x$.

The free energy of the system is assumed here to be expressed by the sum of two kinds of free energy; one associated with the internal degrees of freedom or intrasegmental interactions including the interactions related to the chain conformations, and the other associated with the external degrees of freedom or intersegmental interactions. The former is considered to be independent of volume and depends on the state of chain conformations and temperature. Hence it can be regarded as a function of $\lambda, a, b$ and $T$. On the other hand, the latter (free energy) should depend upon the hole fraction, the mean distance between nearest neighbor segments (the cell dimension) and temperature. Therefore, it can be regarded as a function of $\tilde{V}, l_{i}^{+}$, and $T$. The free energy may not directly depend on $m_{i}$ but on $\tilde{V}$. Such an assumption, that the two kinds of free energy related respectively to chain conformations and liquidlike properties are expressed separately, was adopted in a previous paper ${ }^{1}$ and was also introduced in the statistical theory of rubber elasticity. ${ }^{3}$

The form of eq 10 is now introduced also in the present theory. Then, under the above as- 
Equation of State and Glass Transition of Stretched Network Polymers

sumption the free energy of the system is expressed in the form;

$$
\begin{aligned}
F= & F_{1}(\lambda, b, a, T)+F_{2}\left(\tilde{V}, l_{1,2,3}^{+}, T\right) \\
= & U_{1}{ }^{\mathrm{c}}(\lambda, b)-T S_{1}{ }^{\mathrm{c}}(\lambda, b)+U_{1}{ }^{a}(\boldsymbol{a}, T)-T S_{1}{ }^{a}(a, T) \\
& +U_{2}{ }^{\mathrm{c}}(\tilde{V})-T S_{2}{ }^{\mathrm{c}}(\tilde{V})+U_{2}{ }^{+}\left(l_{1,2,3}^{+}, T\right) \\
& -T S_{2}{ }^{+}\left(l_{1,2,3}^{+}, T\right)
\end{aligned}
$$

where the subscripts 1 and 2 refer to intra- and inter-segmental interactions, respectively.

\section{Equation of State}

The equation of state for simple elongation can be derived in the conventional manner:

$$
\begin{aligned}
& V(\sigma-P)=\left(\partial F / \partial \ln L_{1}\right)_{T, L_{2,3}} \\
& -V P=\left(\partial F / \partial \ln L_{2}\right)_{T, L_{3,1}}
\end{aligned}
$$

where $\sigma$ is the tensile stress, the tensile force per unit area. In the case of simple elongation, since the total differential of $F$ is written, corresponding to eq $1^{\prime}$, as $\mathrm{d} F=-S \mathrm{~d} T-P \mathrm{~d} \dot{V}+f \mathrm{~d} L_{1}-\sum_{i=1}^{m} A_{i} \mathrm{~d} y_{i}$ ( $f$, tensile force), the affinity $A_{i}$ is defined as $A_{i}=-\left(\partial F / \partial y_{i}\right)_{T, V, L_{1}, y_{j \neq i}}$ with $L_{2}=L_{3}$, where $y=$ $\left\{\lambda_{1}, \lambda_{2}, \lambda_{3}, m_{1}, m_{2}, m_{3}, b\right\}$. Then, in the liquid state, the equilibrium conditions, $A_{i}=0$, are satisfied, which are written, with the aid of eq $23-26,32$ and 33 , as

$$
\begin{aligned}
A_{\lambda_{i}} & \equiv-\left(\partial F / \partial \lambda_{i}\right)_{T, V, L_{1}, \lambda_{j \neq i}, m_{1,2,3}, b} \\
& =-\left(\partial F / \partial \lambda_{i}\right)_{\tilde{V}, l_{1,2,3}^{+}, \lambda_{j \neq i}, a_{j \neq i}, b, T} \\
& =0 \\
A_{m_{i}} & \equiv-\left(\partial F / \partial m_{i}\right)_{T, V, L_{1}, \lambda, m_{j \neq i}, b} \\
& =-\left(\tilde{V} / m_{i}\right)(\partial F / \partial \tilde{V})_{l_{j \neq i}^{+}, \lambda, a, b, T}^{+} \\
& =\left(l_{i}^{+} / m_{i}\right)\left(\partial F / \partial l_{i}^{+}\right)_{l_{j \neq i}^{+}, \lambda, a, b, T}^{+} \\
& =0
\end{aligned}
$$

Using the relations of eq $23-27$ and eq 32 , eq 36 yields

$$
\begin{aligned}
& K_{1}^{+}=K_{2}^{+}=K_{3}^{+}=\tilde{K} \\
& K_{1}{ }^{\lambda}=K_{1}{ }^{a}, \quad K_{2}{ }^{\lambda}=K_{2}^{a}=K_{3}^{\lambda}=K_{3}{ }^{a}
\end{aligned}
$$

where

$$
\begin{aligned}
& K_{i}^{+}=\left(\partial F / \partial \ln l_{i}^{+}\right)_{\tilde{V}, \lambda, l_{j \neq i}^{+}, a, b, T} \\
& \tilde{K}=(\partial F / \partial \ln \tilde{V})_{l_{1,2,3}^{+}, \lambda, a, b, T} \\
& K_{i}{ }^{\lambda}=\left(\partial F / \partial \ln \lambda_{i}\right)_{\tilde{V}, l_{1,2,3}^{+}, \lambda_{j \neq i} a, b, T} \\
& K_{i}{ }^{a}=\left(\partial F / \partial \ln a_{i}\right)_{\tilde{V}, l_{1,2,3}^{+}, \lambda, a}^{+} \neq i, b, T
\end{aligned}
$$

The eq 37 gives

Polymer J., Vol. 4, No. 3, 1973

$$
l_{1}^{+}=l_{2}^{+}=l_{3}^{+}
$$

which indicates that the liquidlike properties are isotropic in the liquid state in the present treatment. The equation of state in the liquid state is then expressed, from eq $34,35,37$, and 38 , as

$$
\begin{aligned}
& V(\sigma-P)=K_{1}{ }^{2}+K_{1}{ }^{+} \\
& -V P=K_{2}{ }^{2}+K_{2}^{+}
\end{aligned}
$$

with $K_{i}{ }^{2}=K_{i}^{a}$ and $K_{1}^{+}=K_{2}^{+}=K_{3}^{+}=\tilde{K}$. From eq 44 and 45 , the tensile force $f=\sigma V / L_{1}$ is

$$
f=\left(K_{1}{ }^{\lambda}-K_{2}{ }^{\lambda}\right) / L_{1}
$$

On the other hand, in the glassy state $\lambda_{i}, \tilde{V}$, $m_{i}$, and $b$ are kept constant, then it follows, from eq $23-27,32,34$, and 35 with $\mathrm{d} \ln \lambda_{i}=\mathrm{d} \ln \tilde{V}=$ $\mathrm{d} \ln m_{i}=\mathrm{d} \ln b=0$, that

$$
\begin{aligned}
& V(\sigma-P)=K_{1}^{a}+K_{1}^{+} \\
& -V P=K_{2}^{a}+K_{2}^{+} \\
& f=\left\{\left(K_{1}^{a}-K_{2}^{a}\right)+\left(K_{1}^{+}-K_{2}^{+}\right)\right\} / L_{1}
\end{aligned}
$$

At the glass transition point, $K_{1}^{+}=K_{2}^{+}, K_{i}{ }^{2}=K_{i}^{a}$, and therefore it is seen from eq 46 and 49 that $f$ is continuous at the transition.

From the equation of state one can now evaluate the thermal expansion coefficient, compressibility, Poisson's ratio, and Young's modulus, which will be defined in the following, in terms of the derivatives of free energy and entropy with respect to the parameters involved in the theory:

thermal expansion coefficient;

$$
\begin{aligned}
& \alpha_{1 L}=(\partial \ln V / \partial T)_{P, L_{1}}^{1} \\
& \alpha_{1 L}^{+}=\left(\partial \ln V^{+} / \partial T\right)_{P, L_{1}}^{1} \\
& \alpha_{\mathrm{g} L}=(\partial \ln V / \partial T)_{P, L_{1}}^{\mathrm{g}} \\
& \alpha_{\mathrm{g}}=(\partial \ln V / \partial T)_{P, \sigma=0}^{\mathrm{g}}
\end{aligned}
$$

isothermal compressibility

$$
\begin{aligned}
& \beta_{1 L}=-(\partial \ln V / \partial P)_{T, L_{1}}^{1} \\
& \beta_{1 L}^{+}=-\left(\partial \ln V^{+} / \partial P\right)_{T, L_{1}}^{1} \\
& \beta_{\mathrm{g} L}=-(\partial \ln V / \partial P)_{T, L_{1}}^{\mathrm{g}} \\
& \beta_{\mathrm{g}}=-(\partial \ln V / \partial P)_{T, \sigma=0}^{\mathrm{g}}
\end{aligned}
$$

Poisson's ratio

$$
\begin{aligned}
& \mu_{1}=-\left(\partial \ln L_{2} / \partial \ln L_{1}\right)_{P, T}^{1} \\
& \mu_{\mathrm{g}}=-\left(\partial \ln L_{2} / \partial \ln L_{1}\right)_{P, T}^{\mathrm{g}}
\end{aligned}
$$

Young's modulus

$$
E_{1}=\left(\partial \sigma / \partial \ln L_{1}\right)_{P, T}^{1}
$$




$$
E_{\mathrm{g}}=\left(\partial \sigma / \partial \ln L_{1}\right)_{P, T}^{\mathrm{g}}
$$

The superscripts 1 and $\mathrm{g}$ again indicate the derivatives in the liquid and glassy states respectively. The following derivatives are also defined here:

$$
\begin{aligned}
& K_{i j}^{+}=\left(\partial K_{i}^{+} / \partial \ln l_{j}^{+}\right)_{\tilde{V}, l_{k \neq j}^{+}, T} \\
& \widetilde{K}=(\partial \tilde{K} / \partial \ln \tilde{V})_{l_{1,2,3}+T}^{+} \\
& K_{i j}^{\lambda}=\left(\partial K_{i}^{\lambda} / \partial \ln \lambda_{j}\right)_{\lambda_{k \neq j}, b, a, T} \\
& K_{i j}^{a}=\left(\partial K_{i}^{a} / \partial \ln a_{j}\right)_{\lambda, b, a_{k \neq j}, T}
\end{aligned}
$$

As the results, from eq 44, 45, 47, and 48 with eq 37 and 38 for the liquid state, the following expressions are obtained (The derivations are shown in APPENDIX I).

$$
\begin{aligned}
& \alpha_{1 L}-\alpha_{1 L}^{+}=\left\{\left(\partial S_{2}^{\mathrm{c}} / \partial \ln \tilde{V}\right)-\left(\partial K_{2}{ }^{\lambda} / \partial T\right)_{P, L_{1}}^{1}\right. \\
& \left.-V P \alpha_{1 L}\right\} / \tilde{\widetilde{K}} \\
& \alpha_{1 L}^{+}=-3\left\{\left(\partial K_{2}^{+} / \partial T\right)_{l_{1,2,3}^{+}}+\left(\partial K_{2}{ }^{\lambda} / \partial T\right)_{P, L_{1}}^{1}\right. \\
& \left.+P V \alpha_{1 L}\right\} / \sum_{k=1}^{3} K_{-2 k}^{+} \\
& \alpha_{\mathrm{g} L}=-\left\{\left(\partial K_{2}^{+} / \partial T\right)_{l_{1,2,3}^{+}}+\left(\partial K_{2}^{a} / \partial T\right)_{a_{1,2,3}}\right\} / \\
& \left\{\sum_{k=2}^{3} K_{2 k}^{+} / 2+\sum_{k=2}^{3} K_{2 k}^{a} / 2+P V\right\} \\
& \alpha_{\mathrm{g}}=-\left\{\left(\partial K_{2}^{+} / \partial T\right)_{l_{1,2,3}^{+}}+\left(\partial K_{2}^{a} / \partial T\right)_{a_{1,2,3}}\right\} / \\
& \left\{\sum_{k=1}^{3} K_{2 k}^{+}\left(\partial \ln l_{k}^{+} / \partial \ln V\right)_{P, o=0}^{\mathrm{g}}\right. \\
& \left.+\sum_{k=1}^{3} K_{2 k}^{a}\left(\partial \ln a_{k} / \partial \ln V\right)_{P, \sigma=0}^{\mathrm{g}}+P V\right\} \\
& \beta_{1 L}-\beta_{1 L}^{+}=V\left\{1-\left(\boldsymbol{P}+\boldsymbol{P}^{\lambda}\right) \beta_{1 L}\right\} / \tilde{\widetilde{K}} \\
& \beta_{1 L}^{+}=3 V\left\{1-\left(P+P^{\lambda}\right) \beta_{1 L}\right\} / \sum_{k=1}^{3} K_{2 k}^{+} \\
& \beta_{g L}=\left(\boldsymbol{P}+\boldsymbol{P}^{a}+\sum_{k=2}^{3} K_{2 k}^{+} / 2 V\right)^{-1} \\
& \beta_{\mathrm{g}}=\left\{\boldsymbol{P}+\boldsymbol{P}^{a}+\sum_{k=1}^{3} K_{2 k}^{+}\left(\partial \ln l_{k}^{+} / \partial V\right)_{T, \sigma=0}^{\mathrm{g}}\right\}^{-1} \\
& 1-2 \mu_{1}=\left(\partial \ln V / \partial \ln L_{1}\right)_{T, P}^{1} \\
& =\frac{\left(\partial K_{2}{ }^{\lambda} / \partial \ln L_{1}\right)_{T, P}^{1}\left(3 \tilde{K}+\sum_{k=1}^{3} K_{2 k}^{+}\right)}{\tilde{K} \sum_{k=1}^{3} K_{2 k}^{+}+P V\left(3 \tilde{K}+\sum_{k=1}^{3} K_{2 k}^{+}\right)} \\
& \left(\sum_{k=2}^{3} K_{2 k}^{+} / 2+P^{a} V+P V\right)
\end{aligned}
$$

$$
\begin{aligned}
& E_{\mathrm{g}}=\left\{K_{11}^{a}+K_{11}^{+}-2\left(K_{12}^{a}+K_{12}^{+}\right) \mu_{\mathrm{g}}\right\} / V \\
&-\left(1-2 \mu_{\mathrm{g}}\right)(\sigma-P) \\
& \sum_{k=1}^{3} K_{2 k}^{+}\left(\partial \ln l_{k}^{+} / \partial L_{1}\right)_{T, L}^{1}=\tilde{K}\left(\partial \ln V / \partial L_{1}\right)_{T, P}^{1}
\end{aligned}
$$

where

$$
\begin{aligned}
& P^{\lambda}=\sum_{k=1}^{3} K_{2 k}^{\lambda}\left(\partial \ln \lambda_{k} / \partial V\right)_{T, L_{1}}^{1} \\
& P^{a}=\sum_{k=1}^{3} K_{2 k}^{a}\left(\partial \ln a_{k} / \partial V\right)_{T, L_{1}}^{\mathrm{g}}=\sum_{k=2}^{3} K_{2 k}^{a} / 2 V
\end{aligned}
$$

$\boldsymbol{P}_{0}{ }^{a}=\sum_{k=1}^{3} \boldsymbol{K}_{2 k}^{a}\left(\partial \ln a_{k} / \partial V\right)_{T, \sigma=0}^{\mathrm{g}}$

On the other hand, heat capacity is evaluated as follows.

Heat capacity under constant pressure $C_{p}$ consists of two parts because of the form of free energy eq 33:

$$
C_{p}=C_{p}^{\text {inter }}+C_{p}^{\text {intra }}
$$

where the superscripts inter and intra refer to inter- and intra-segmental interactions. From the definition of $C_{p}$ and eq 23, one has

$$
\begin{aligned}
C_{p 1 L}^{\mathrm{intra}} \equiv & T\left(\partial S_{1} / \partial T\right)_{p, L_{1}}^{1} \\
= & T\left(\partial S_{1}^{\mathrm{c}} / \partial T\right)_{p, L_{1}}+T\left(\partial S_{1}{ }^{a} / \partial T\right)_{p, L_{1}} \\
= & \sum_{k=1}^{3} T\left(\partial S_{1}{ }^{\mathrm{c}} / \partial \ln \lambda_{k}\right)_{b}\left(\partial \ln \lambda_{k} / \partial T\right)_{p, L_{1}}^{1} \\
& +T\left(\partial S_{1}{ }^{\mathrm{c}} / \partial T\right)_{\lambda_{1,2,3}}+\sum_{k=1}^{3} T\left(\partial S_{1}{ }^{a} / \partial \ln a_{k}\right)_{T} \\
& \times\left(\partial \ln a_{k} / \partial T\right)_{p, L_{1}}^{1}+T\left(\partial S_{1}{ }^{a} / \partial T\right)_{a_{1,2,3}}
\end{aligned}
$$

where it was noticed that $b$ is a function of $T$ alone. Similarly,

* The heat transferred to the material in an infinitesimal process, $\delta Q$, is expressed as ${ }^{2}$

$$
\delta Q=T \mathrm{~d} S-\sum_{i} A_{i} \mathrm{~d} y_{i}
$$

In the liquid state, an equilibrium state, $A_{i}=0$, therefore, one has $\delta Q=T \mathrm{~d} S$. On the other hand, in the glassy state, $\mathrm{d} y_{i}=0$ is assumed here, accordingly $\delta Q=T \mathrm{~d} S$ also holds. Hence, the heat capacity is evaluated as $T(\mathrm{~d} S / \mathrm{d} T)$ even in the glassy state where $A_{i} \neq 0$. Only in an irreversible process where the change of $y_{i}$ occurs in a non-equilibrium state $\left(\mathrm{d} y_{i} \neq\right.$ 0 and $A_{i} \neq 0$ ), one has $\delta Q \neq T \mathrm{~d} S$ and $T \mathrm{~d} S-\delta Q=$ $\sum_{i} A_{i} \mathrm{~d} y_{i}>0$, when thermal and pressure equilibriums are assumed as is done in this paper. 
Equation of State and Glass Transition of Stretched Network Polymers

$$
\begin{aligned}
C_{p g L}^{\mathrm{intra}} \equiv & T\left(\partial S_{1} / \partial T\right)_{p, L_{1}}^{\mathrm{g}}=T\left(\partial S_{1}{ }^{a} / \partial T\right)_{p, L_{1}}^{\mathrm{g}} \\
= & T \sum_{k=1}^{3}\left(\partial S_{1}{ }^{a} / \partial \ln a_{k}\right)_{T}\left(\partial \ln a_{k} / \partial T\right)_{p, L_{1}}^{\mathrm{g}} \\
& +T\left(\partial S_{1}{ }^{a} / \partial T\right)_{a_{1,2,3}} \\
= & T \alpha_{g L} \sum_{k=2}^{3}\left(\partial S_{1}{ }^{a} / \partial \ln a_{k t}\right)_{T} / 2+T\left(\partial S_{1}{ }^{a} / \partial T\right)_{a_{1,2,3}}
\end{aligned}
$$

In the derivation of eq 84 from eq $83,\left(\partial \ln a_{1} /\right.$ $\partial T)_{p, L_{1}}^{\mathrm{g}}=0$ and $\left(\partial \ln a_{2} / \partial T\right)_{p, L_{1}}^{\mathrm{g}}=\left(\partial \ln a_{3} / \partial T\right)_{p, L_{1}}^{\mathrm{g}}=$ $\alpha_{g L}$ were used. On the other hand, one may derive from the form of expression for $S$ in eq 33

$$
\begin{aligned}
C_{p g}^{\mathrm{intra}} \equiv & T\left(\partial S_{1} / \partial T\right)_{p, \sigma=0}^{\mathrm{g}}=T\left(\partial S_{1}{ }^{a} / \partial T\right)_{p, \sigma=0}^{\mathrm{g}} \\
= & T \sum_{k=1}^{3}\left(\partial S_{1}{ }^{a} / \partial \ln a_{k}\right)_{T}\left(\partial \ln a_{k} / \partial T\right)_{p, \sigma=0}^{\mathrm{g}} \\
& +T\left(\partial S_{1}{ }^{a} / \partial T\right)_{a_{1,2,3}}
\end{aligned}
$$

Heat capacity $C_{p}^{\text {ir.ter }}$ associated with intersegmental interactions is also derived as follows in the same way as the above:

$$
\begin{aligned}
& C_{p 1 L}^{\text {inter }}=T\left(\partial S_{2}{ }^{\mathrm{c}} / \partial \ln \tilde{V}\right)(\partial \ln \tilde{V} / \partial T)_{p, L_{1}}^{1} \\
& +T \sum_{k=1}^{3}\left(\partial S_{2}^{+} / \partial \ln l_{k}^{+}\right)_{T, l_{i \neq k}^{+}}^{+} \\
& \times\left(\partial \ln l_{k}^{+} / \partial T\right)_{p, L_{1}}^{1}+T\left(\partial S_{2}^{+} / \partial T\right)_{l_{1,2,3}^{+}} \\
& =T\left(\alpha_{1 L}-\alpha_{1 L}^{+}\right)\left(\partial S_{2}{ }^{\mathrm{c}} / \partial \ln \tilde{V}\right) \\
& +T \alpha_{1 L} \sum_{k=1}^{3}\left(\partial S_{2}^{+} / \partial \ln l_{k}^{+}\right)_{T, l_{i \neq k}^{+} / 3} \\
& +T\left(\partial S_{2}^{+} / \partial T\right)_{l_{1,2,3}^{+}} \\
& C_{p 1 L}^{+} \equiv T\left(\partial S_{2}^{+} / \partial T\right)_{p, L_{1}}^{1} \\
& =T \alpha_{1 L} \sum_{k=1}^{3}\left(\partial S_{2}^{+} / \partial \ln l_{k}^{+}\right)_{T, l_{i \neq k}^{+} / 3} \\
& +T\left(\partial S_{2}^{+} / \partial T\right)_{l_{1,2,3}^{+}} \\
& C_{p 1 L}^{\text {inter }}-C_{p 1 L}^{+}=T\left(\alpha_{1 L}-\alpha_{1 L}^{+}\right)\left(\partial S_{2}{ }^{c} / \partial \ln \tilde{V}\right) \\
& C_{\mathrm{g} L}^{1 \text { in ter }}=T \sum_{k=1}^{3}\left(\partial S_{2}^{+} / \partial \ln l_{k}^{+}\right)_{T, l_{i \neq k}^{+}}^{+}\left(\partial \ln l_{k}^{+} / \partial T\right)_{p, L_{1}}^{\mathrm{g}} \\
& +T\left(\partial S_{2}^{+} / \partial T\right)_{l_{1,2,3}^{+}} \\
& =T \alpha_{\mathrm{g} L} \sum_{k=2}^{3}\left(\partial S_{2}^{+} / \partial \ln l_{k}^{+}\right)_{T, l_{i \neq k}^{+} / 2} \\
& +T\left(\partial S_{2}^{+} / \partial T\right)_{l_{1,2,3}^{+}}^{+} \\
& C_{p \mathrm{~g}}^{\mathrm{inter}}=T \sum_{k=1}^{3}\left(\partial S_{2}^{+} / \partial \ln l_{k}^{+}\right)_{T, l_{i \neq k}^{+}} \\
& \times\left(\partial \ln l_{k}^{+} / \partial T\right)_{p, a=0}^{\mathrm{g}}+T\left(\partial S_{2}^{+} / \partial T\right)_{l_{1,2,3}^{+}}
\end{aligned}
$$

\section{Approximate Expressions}

Further considerations will be made under those approximations which are reasonable as follows.
From the condition of equiliblium, eq 38 , one obtains

$$
\begin{aligned}
& \left(\partial U_{1}^{a} / \partial \ln a_{i}\right)_{a_{j \neq i}, T}-T\left(\partial S_{1}{ }^{a} / \partial \ln a_{i}\right)_{a_{j \neq i}, T} \\
& \quad=\left(\partial U_{1}^{\mathrm{c}} / \partial \ln \lambda_{i}\right)_{\lambda_{j \neq i}, T}-T\left(\partial S_{1}^{\mathrm{c}} / \partial \ln \lambda_{i}\right)_{\lambda_{j \neq i}, T} \\
& \quad=K_{i}{ }^{2}
\end{aligned}
$$

The first part of the left hand side of this equation indicates the stress, arising from the variation of bond length and small deviation of rotational angles from the stable positions, which may be considered to be almost the energetic force, i.e., $\left(\partial U_{1}^{a} / \partial \ln a_{i}\right)_{a_{j \neq i}, T} \gg T\left(\partial S_{1}{ }^{a} / \partial \ln a_{i}\right)_{a_{j \neq i}, T}$. On the other hand, $T\left(\partial S_{1}^{\mathrm{c}} / \partial \ln \lambda_{i}\right)_{\lambda_{j \neq i}, T}$ is a dominant part in $K_{i}{ }^{\lambda}$ (or the same order as $K_{i}^{\lambda}$ ), where $K_{i}^{\lambda}$ designates the stress arising from the change in trans-gauche conformation. Therefore, it is assumed here that

$$
T\left(\partial S_{1}{ }^{c} / \partial \ln \lambda_{i}\right)_{\lambda_{j \neq i}, T} \gg T\left(\partial S_{1}{ }^{a} / \partial \ln a_{i}\right)_{a_{j \neq i}, T}
$$

or

$$
\left(\partial K_{i}^{\lambda} / \partial T\right)_{\lambda_{1,2,3}} \gg\left(\partial K_{i}^{a} / \partial T\right)_{a_{1,2,3}}
$$

On the other hand, eq 38 yields

$$
\sum_{k=1}^{3} K_{\imath k}^{a}\left(\mathrm{~d} \ln a_{k}\right)_{T}{ }^{1}=\sum_{k=1}^{3} K_{i k}^{\lambda}\left(\mathrm{d} \ln \lambda_{k}\right)_{T}{ }^{1}
$$

Accordingly, it may be considered that

$$
\begin{aligned}
& \left(\partial \ln a_{k} / \partial \ln L_{1}\right)_{T, P}^{1} /\left(\partial \ln \lambda_{i} / \partial \ln L_{1}\right)_{T, P}^{1} \\
& \quad=\mathrm{O}\left(K_{\imath k}^{\lambda} / K_{i k}^{a}\right) \ll 1
\end{aligned}
$$

because the modulus $K_{i k}^{a}$ for the stress $K_{i}^{a}$ may be much larger than $K_{i k}^{\lambda}$, than for the stress $K_{i}^{\lambda}$.

In the present treatment the stretching of the network polymer in the liquid state under atmospheric pressure is considered, excluding the stretching in the glassy state with the stress larger than that of the stretching in the liquid state. The following conditions may then be satisfied, because $\boldsymbol{P}$ and $\sigma$ are much smaller than the internal pressure $P_{\mathrm{i}}\left[\equiv(\partial U / \partial V)_{T} \equiv T \alpha / \beta-P\right]$ and the bulk modulus $\left(\beta^{-1}\right)\left[\boldsymbol{P}_{\mathrm{i}}=\mathbf{O}\left(10^{3} \mathrm{~atm}\right), \beta^{-1}=\mathbf{O}\left(10^{5} \mathrm{~atm}\right)\right]$, as seen from eq $66,67,70,71$, and 72 with $P_{\mathrm{i}} \cong$ $T(\alpha / \beta)$ and eq 94

$$
\begin{aligned}
& P, P^{\lambda}, \sigma \ll P^{a}, P_{0}{ }^{a} \ll \mathrm{O}\left(\beta^{-1}\right)=\tilde{K}, K_{i k}^{+} \\
& T\left(\partial K_{i}{ }^{\lambda} / \partial T\right)_{P, L_{1}}^{1}=\mathrm{O}(\sigma) \ll \mathrm{O}\left(P_{\mathrm{i}}\right) \\
&=T\left(\partial S_{2}{ }^{\mathrm{c}} / \partial \ln \tilde{V}\right), T\left(\partial K_{i}^{+} / \partial T\right)_{l_{1,2,3}^{+}}
\end{aligned}
$$

In the case considered here, the anisotropy in 
the liquid-like properties is very small and may be ignored even in the glassy state; that is,

$\left(\partial \ln l_{k}{ }^{+} / \partial \ln V\right)_{T, \sigma=0}^{\mathrm{g}}=1 / 3$ for $k=1,2,3$

$\left(\partial \ln l_{k}^{+} / \partial T\right)_{p, a=0}^{\mathrm{g}}=\alpha_{\mathrm{g}} / 3 \quad$ for $k=1,2,3$

$K_{i i}^{+}=K_{j j}^{+}, \quad K_{12}^{+}=K_{21}^{+}=K_{23}^{+}=K_{32}^{+}=K_{31}^{+}=K_{13}^{+}$

Then, one has from eq 70 and 71 with the aid of eq 95 ,

$$
\begin{aligned}
& \beta_{1 L}-\beta_{1 L}^{+}=V / \tilde{K} \\
& \beta_{1 L}^{+}=3 V / \sum_{k=1}^{3} K_{2 k}^{+}
\end{aligned}
$$

On the other hand, eq 72 and 73 are expressed from eq 95 and 97 as

$$
\begin{aligned}
& \beta_{\mathrm{g} L}=2 V / \sum_{k=2}^{3}\left(K_{2 k}^{+}+K_{2 k}^{a}\right) \cong 2 V / \sum_{k=2}^{3} K_{2 k}^{+} \\
& \beta_{\mathrm{g}}=3 V / \sum_{k=1}^{3}\left(K_{2 k}^{+}+K_{2 k}^{a}\right) \cong 3 V / \sum_{k=1}^{3} K_{2 k}^{+}
\end{aligned}
$$

With the use of eq 95 , eq 66 and 67 reduce to

$$
\begin{aligned}
& \alpha_{1 L}-\alpha_{1 L}^{+}=\left(\partial S_{2}^{\mathrm{c}} / \partial \ln \tilde{V}\right) / \widetilde{\tilde{K}} \\
& \alpha_{i L}^{+}=-3\left(\partial K_{2}^{+} / \partial T\right)_{l_{1,2,3}^{+}} /\left(\sum_{k=1}^{3} K_{2 k}^{+}\right)
\end{aligned}
$$

Under the condition of eq $92^{\prime}, 95$, and 97 , eq 68 and 69 yield

$$
\begin{aligned}
\alpha_{\mathrm{g} L}= & -2\left\{\left(\partial K_{2}^{+} / \partial T\right)_{l_{1,2,3}^{+}}\right. \\
& \left.+\left(\partial K_{2}^{a} / \partial T\right)_{a_{1,2,3}}\right\} / \sum_{k=2}^{3}\left(K_{2 k}^{+}+K_{2 k}^{a}\right) \\
\cong & -2\left(\partial K_{2}^{+} / \partial T\right)_{l_{1,2,3}^{+}} / \sum_{k=2}^{3} K_{2 l}^{+} \\
\alpha_{\mathrm{g}}= & -3\left\{\left(\partial K_{2}^{+} / \partial T\right)_{l_{1,2,3}^{+}}^{+}+\left(\partial K_{2}^{a} / \partial T\right)_{a_{1,2,3}}\right\} / \\
& \sum_{k=1}^{3}\left(K_{2 k}^{+}+K_{2 l k}^{a}\right) \\
= & -3\left(\partial K_{2}^{+} / \partial T\right)_{l_{1,2,3}^{+}} / \sum_{k=1}^{3} K_{2 k}^{+}
\end{aligned}
$$

Furthermore the approximation of eq 95 makes eq 74 reduce to

$$
\begin{aligned}
& \left(\partial \ln V / \partial \ln L_{1}\right)_{T, P}^{1} \\
& \quad=-\left(\partial K_{2}{ }^{2} / \partial \ln L_{1}\right)_{T, P}^{1}\left\{3 / \sum_{k=1}^{3} K_{2 k}^{+}+\widetilde{K}^{-1}\right\}
\end{aligned}
$$

and makes eq 75 deduce to

$$
\left(\partial \ln V / \partial \ln L_{1}\right)_{T}^{\mathrm{g}, P}=1-\left(2 K_{21}^{+} / \sum_{k=2}^{3} K_{2 k}^{+}\right)
$$

The inequality of eq 95 also simplifies eq 76 as

$$
E_{\mathrm{g}}=\left\{K_{11}^{+}-2 K_{12}^{+} \mu_{\mathrm{g}}\right\} / V
$$

From eq 103, 109, and 110 with eq 99 , one can derive the relation;

$$
3\left(1-2 \mu_{\mathrm{g}}\right)=E_{\mathrm{g}} \beta_{\mathrm{g}}
$$

This relation is well known for isotropic substances.

Under the approximation of eq 98 , eq 90 is expressed as

$$
\begin{aligned}
C_{p \mathrm{~g}}^{\text {inter }}= & T \alpha_{\mathrm{g}} \sum_{k=1}^{3}\left(\partial S_{2}^{+} / \partial \ln l_{k}^{+}\right)_{T, l_{i \neq k}^{+} / 3} \\
& +T\left(\partial S_{2}^{+} / \partial T\right)_{l_{1,2,3}^{+}}^{+}
\end{aligned}
$$

Finally one can obtain the following relations at the glass transition from eq $101,103,105,107^{\prime}$ 87, and 112:

$$
\begin{aligned}
& \beta_{1 L}^{+}=\beta_{\mathrm{g}} \\
& \alpha_{1 L}^{+}=\alpha_{\mathrm{g}} \\
& C_{p 1 L}^{+}=C_{p \mathrm{~g}}^{\text {inter }}
\end{aligned}
$$

Then, it follows from eq 88, 104, and 100 that

$$
T V(\Delta \alpha)^{2} / \Delta C_{p}^{\text {inter }} \Delta \beta=1
$$

where

$$
\begin{aligned}
& \Delta C_{p}^{\text {inter }} \equiv C_{p 1 L}^{\text {inter }}-C_{p 1 L}^{+}=C_{p 1 L}^{\text {inter }}-C_{p \mathrm{~g}}^{\text {inter }} \\
& \Delta \alpha \equiv \alpha_{1 L}-\alpha_{1 L}^{+}=\alpha_{1 L}-\alpha_{\mathrm{g}} \\
& \Delta \beta \equiv \beta_{1 L}-\beta_{1 L}^{+}=\beta_{1 L}-\beta_{\mathrm{g}}
\end{aligned}
$$

Similarly one has from eq $100,103,104$, and $107^{\prime}$ (or 101 and 105)

$$
\begin{aligned}
& \left(\partial S_{2}{ }^{\mathrm{c}} / \partial \ln \tilde{V}\right)=V \Delta \alpha / \Delta \beta \\
& \tilde{\tilde{K}}=V / \Delta \beta \\
& \left(\partial K_{2}^{+} / \partial T\right)_{l_{1,2,3}^{+}}^{+}=\left(\partial S_{2}^{+} / \partial \ln l_{2}^{+}\right)_{l_{i \neq 2}^{+}}^{+} \\
& =V \alpha_{1 L}^{+} / \beta_{1 L}^{+}=V \alpha_{\mathrm{g}} / \beta_{\mathrm{g}}
\end{aligned}
$$

From eq 23, 27, 43, 77, 100, and 101, we have

$$
\begin{aligned}
& \left(\partial \ln l_{i}^{+} / \partial \ln V\right)_{T, L_{1}}^{1} \\
& \quad=\left(\partial \ln l_{i}^{+} / \partial \ln V\right)_{T, P}^{1}=\beta_{1 L}^{+} / 3 \beta_{1 L} \\
& \left(\partial \ln \tilde{V} / \partial \ln l_{i}^{+}\right)_{T, L_{1}}^{1} \\
& \quad=\left(\partial \ln V / \partial \ln l_{i}^{+}\right)_{T, P}^{1}=3 \Delta \beta / \beta_{1 L}^{+}
\end{aligned}
$$

It is finally pointed out that in the present approximations the derivatives under constant length in the liquid state, $\alpha_{1 L}, \beta_{1 L}$, and $C_{p 1 L}$, depend little on $L_{1}$ and become equal to those at $\sigma=0$. (APPENDIX II) Therefore, $\alpha_{p 1 L}^{+}, \beta_{1 L}^{+}$, and $C_{p 1 L}^{+}$, accordingly $\alpha_{\mathrm{g}}, \beta_{\mathrm{g}}$ and $C_{p \mathrm{~g}}$ because of eq 113- 
Equation of State and Glass Transition of Stretched Netwark Polymers

115 , are also regarded as independent of the length in the present treatment.

\section{Expressions for Stretch Effect on Glass Transition Temperature}

First, the configurational thermodynamic quantity $S^{\mathrm{c}}\left(\right.$ or $\left.U^{\mathrm{c}}\right)$ is defined as a part of $S($ or $U$ ) which is frozen-in in the glassy states: (cf. eq 10)

$$
\begin{aligned}
& S^{\mathrm{c}}={S_{1}}^{\mathrm{c}}(\lambda, b)+{S_{2}}^{\mathrm{c}}(\tilde{V}) \\
& U^{\mathrm{c}}=U_{1}{ }^{\mathrm{c}}(\lambda, b)+U_{2}{ }^{\mathrm{c}}(\tilde{V})
\end{aligned}
$$

Putting $Y_{s}=T, Y_{t}=L_{1}$, and $X=S^{\mathrm{c}}$ in eq 13 , we have

$$
\begin{aligned}
\left(\partial T / \partial L_{1}\right)_{\mathrm{g}}= & \left\{-\left(\partial S^{\mathrm{c}} / \partial L_{1}\right)_{T, P}^{1}\right. \\
& \left.+\left(\partial S^{\mathrm{c}} / \partial L_{1}\right)_{\mathrm{g}}\right\} /\left(\partial S^{\mathrm{c}} / \partial T\right)_{L_{1}, P}^{1}
\end{aligned}
$$

The term $\left(\partial S^{\mathrm{c}} / \partial L_{1}\right)_{T, P}^{1}$ is expressed with the aid of eq $92,121,122$, and $(\partial P / \partial T)_{V, L_{1}}^{1} \equiv \alpha_{1 L} / \beta_{1 L}$, as

$$
\begin{aligned}
&\left(\partial S^{\mathrm{c}} / \partial L_{1}\right)_{T, P}^{1} \\
&=\left(\partial S / \partial L_{1}\right)_{T, P}^{1}-\left\{\left(\partial S_{2}^{+} / \partial L_{1}\right)_{T, P}+\left(\partial S_{1}^{a} / \partial L_{1}\right)_{T, P}^{1}\right\} \\
&=\left(\partial S / \partial L_{1}\right)_{T, P}^{1}+\left(\partial V / \partial L_{1}\right)_{T, P}^{1}(\partial P / \partial T)_{V, L_{1}}^{1} \\
&-\left\{\left(\partial S_{2}^{+} / \partial \ln l_{i}^{+}\right)_{T}\left(\partial \ln l_{i}^{+} / \partial \ln V\right)_{T, P}^{1}\right. \\
& \times\left(\partial \ln V / \partial L_{1}\right)_{T, P}^{1} \\
&\left.+\left(\partial S_{1}{ }^{a} / \partial \ln a_{i}\right)_{T}\left(\partial \ln a_{i} / \partial L_{1}\right)_{T, P}^{1}\right\} \\
&=-f_{s} / T+\Delta \alpha\left(\partial V / \partial L_{1}\right)_{T, P}^{1} / \beta_{1 L}
\end{aligned}
$$

Here, $f_{s}$ is derived as

$$
\begin{aligned}
f_{s} & \equiv T\left(\partial S / \partial L_{1}\right)_{T, V}^{1} \\
& \equiv T\left(\partial S / \partial L_{1}\right)_{T, P}^{1}+T(\partial P / \partial T)_{V, L_{1}}^{1}\left(\partial V / \partial L_{1}\right)_{T, P}^{1}
\end{aligned}
$$

On the other hand, $\left(\partial S^{\mathrm{c}} / \partial T\right)_{P, L_{1}}^{1}$ is evaluated with eq $82,85,86,87$, and 92 , as

$$
\begin{aligned}
\left(\partial S^{\mathrm{c}} / \partial T\right)_{P, L_{1}}^{1} & =C_{p 1 L}^{\text {inter }}-C_{p 1 L}^{+}+C_{p 1 L}^{\text {intra }}-C_{p \mathrm{~g}}^{\text {intra }} \\
& =\Delta C_{p}^{\text {inter }}+\Delta C_{p}^{\text {intra }} \\
& \equiv \Delta C_{p}
\end{aligned}
$$

Accordingly, from eq 126,128 , and $130^{\prime}$, one has

$$
\begin{aligned}
\left(\partial T / \partial L_{1}\right)_{\mathrm{g}}= & \left\{f_{s}-T \Delta \alpha\left(\partial V / \partial L_{1}\right)_{T, P}^{1} / \beta_{1 L}\right\} / \Delta C_{p} \\
& +\left(\partial S^{\mathbf{c}} / \partial L_{1}\right)_{\mathrm{g}} / \Delta C_{p}
\end{aligned}
$$

With the same process as the above derivation in the case of $X=S^{\mathrm{c}}$, one can obtain the expression for $X=U^{\mathrm{c}}$ as follows.

$$
\begin{aligned}
\left(\partial T / \partial L_{1}\right)_{\mathrm{g}}= & \left\{-\left(\partial U^{\mathrm{c}} / \partial L_{1}\right)_{T, P}^{1}\right. \\
& \left.+\left(\partial U^{\mathrm{c}} / \partial L_{1}\right)_{\mathrm{g}}\right\} /\left(\partial U^{\mathrm{c}} / \partial T\right)_{L_{1}, P}^{1} \\
\left(\partial U^{\mathrm{c}} / \partial L_{1}\right)_{T, P}^{1}= & f_{\mathrm{e}}+T \Delta \alpha\left(\partial V / \partial L_{1}\right)_{T, P}^{1} / \beta_{1 L}
\end{aligned}
$$

$$
\left(\partial U^{\mathrm{c}} / \partial T\right)_{L_{1}, P}^{1}=\Delta C_{p}
$$

where

$$
f_{\mathrm{e}} \equiv\left(\partial U / \partial L_{1}\right)_{T, V}^{1}
$$

Then, substituting eq 133 and 134 into eq 132 , one has

$$
\begin{aligned}
\left(\partial T / \partial L_{1}\right)_{\mathrm{g}}= & \left\{-f_{\mathrm{e}}-T \Delta \alpha\left(\partial V / \partial L_{1}\right)_{T, P}^{1} / \beta_{1 L}\right\} / \Delta C_{p} \\
& +\left(\partial U^{\mathrm{c}} / \partial L_{1}\right)_{\mathrm{g}} / \Delta C_{p}
\end{aligned}
$$

On the other hand, choosing the variables in eq 13 as $Y_{s}=T, Y_{t}=L_{1}$, and $X=\tilde{V}$, one has with the use of eq 117, 122, and 123 ,

$$
\begin{aligned}
\left(\partial T / \partial L_{1}\right)_{\mathrm{g}}= & \left\{-\left(\partial \ln \tilde{V} / \partial L_{1}\right)_{T, P}^{1}\right. \\
& \left.+\left(\partial \ln \tilde{V} / \partial L_{1}\right)_{\mathrm{g}}\right\} /(\partial \ln \tilde{V} / \partial T)_{L_{1}, P} \\
= & -(\Delta \beta / \Delta \alpha)\left(\partial \ln V / \partial L_{1}\right)_{T, P} / \beta_{1 L} \\
& +\left(\partial \ln \tilde{V} / \partial L_{1}\right)_{\mathrm{g}} / \Delta \alpha
\end{aligned}
$$

Specific Volume and Enthalpies of the Glasses Having Histories of Stretching

Differences in specific volume and enthalpy between two glasses having different histories of stretching compared at the state $(T, \sigma=0)$ are designated as

$$
\begin{aligned}
& \Delta_{\mathrm{g}} \ln V=\ln V(\text { Glass } \mathrm{II})-\ln V(\text { Glass I) } \\
& \Delta_{\mathrm{g}} H=H(\text { Glass II })-H(\text { Glass I })
\end{aligned}
$$

Here, it is provided that the reference glass (Glass I) has been formed at the state $\left(T_{\mathrm{g} 0}, L_{10}, \sigma=0\right)$, and that Glass II has been formed at the state $\left(T_{\mathrm{g}}, L_{1}, \sigma=\sigma_{\mathrm{g}}\right)$. From eq 23 and $27, \Delta_{\mathrm{g}} \ln V$ is separated into two parts:

$$
\Delta_{\mathrm{g}} \ln V=\Delta_{\mathrm{g}} \ln \tilde{V}+\Delta_{\mathrm{g}} \ln V^{+}
$$

The first term $\Delta_{\mathrm{g}} \ln \tilde{V}$ is given from eq 137 as

$$
\Delta_{\mathrm{g}} \ln \tilde{V}=\left(\Delta \beta / \beta_{1 L}\right)(\Delta \ln V)^{1}+\Delta \alpha \Delta T_{\mathrm{g}}
$$

since $\tilde{V}$ is invariable once the glass is formed, where $(\Delta \ln V)^{1}$ is the volume delatiation with stretching in the liquid state, i.e.,

$\int_{L_{10}}^{L_{1}}\left(\partial \ln V / \partial L_{1}\right)_{T, P}^{1} \mathrm{~d} L_{1}, \quad$ and $\quad \Delta T_{\mathrm{g}}=T_{\mathrm{g}}-T_{\mathrm{g} 0}$.

As described in the preceding section, the anisotropy in the liquid-like properties is disregarded here, the dependence of $\alpha_{\mathrm{g}}$ on $L_{1}$ is also neglected. Hence, the second term $\Delta_{\mathrm{g}} \ln V^{+}$in eq 140 is expressed as

$\Delta_{\mathrm{g}} \ln V^{+}=\left(\Delta \ln V^{+}\right)^{1}+\int_{\sigma_{\mathrm{g}}}^{0}\left(\partial \ln V^{+} / \partial \sigma\right)_{P, T}^{\mathrm{g}} \mathrm{d} \sigma$ 
Since the integrand of the second term in eq 142 is evaluated from eq 111 as

$$
\begin{aligned}
\left(\partial \ln V^{+} / \partial \sigma\right)_{P, T}^{\mathrm{g}} \mathrm{d} \sigma \\
\quad=(\partial \ln V / \partial \sigma)_{P, T}^{\mathrm{g}} \mathrm{d} \sigma \\
\quad=\left(\partial \ln V / \partial \ln L_{1}\right)_{P, T}^{\mathrm{g}}\left(\partial \ln L_{1} / \partial \sigma\right)_{P, T}^{\mathrm{g}} \mathrm{d} \sigma \\
\quad=\left(1-2 \mu_{\mathrm{g}}\right) E_{\mathrm{g}}{ }^{-1} \mathrm{~d} \sigma=\beta_{\mathrm{g}} \mathrm{d} \sigma / 3,
\end{aligned}
$$

we have

$$
\Delta_{\mathrm{g}} \ln V^{+}=\left(\beta_{\mathrm{g}} / \beta_{1 L}\right)(\Delta \ln V)^{\mathrm{I}}-\beta_{\mathrm{g}} \sigma_{\mathrm{g}} / 3
$$

Accordingly, one obtains from eq 140, 141, and 143

$$
\Delta_{\mathrm{g}} \ln V=\Delta \alpha \Delta T_{\mathrm{g}}+(\Delta \ln V)^{1}-\beta_{\mathrm{g}} \sigma_{\mathrm{g}} / 3
$$

On the other hand, with the aid of eq 33 and 124 , the enthalpy difference $\Delta_{\mathrm{g}} H$ is expressed as follows under the approximations of eq 95 and 94 , and disregarding the anisotropy in the liquidlike properties:

$$
\begin{aligned}
\partial \Delta_{\mathrm{g}} H / \partial L_{1}= & \left(\partial \Delta_{\mathrm{g}} U^{\mathrm{c}} / \partial L_{1}\right)+\left(\partial \Delta_{\mathrm{g}} U_{1}^{a} / \partial L_{1}\right) \\
& +\left(\partial \Delta_{\mathrm{g}} U_{2}{ }^{+} / \partial L_{1}\right)+P\left(\partial \Delta_{\mathrm{g}} V / \partial L_{1}\right) \\
\cong & \left(\partial U^{\mathrm{c}} / \partial L_{1}\right)_{\mathrm{g}}+\left(\partial U_{2}^{+} / \partial \ln l_{2}^{+}\right)_{l_{\imath \neq 2}^{+}}^{+} \\
& \times\left(\partial \Delta_{\mathrm{g}} \ln V^{+} / \partial L_{1}\right)
\end{aligned}
$$

Since $\left(\partial U_{2}^{+} / \partial \ln l_{2}^{+}\right)_{l_{\imath \neq 2}^{+}}^{+} \cong T\left(\partial S_{2}^{+} / \partial \ln l_{2}^{+}\right)_{i \neq 2}^{+}$because of eq 29 with eq 95 , eq 145 reduces by the use of eq 136, 121, and 143, to

$$
\begin{aligned}
\partial \Delta_{\mathrm{g}} H / \partial L_{1}= & f_{\mathrm{e}}+\Delta C_{p}\left(\partial T / \partial L_{1}\right)_{\mathrm{g}}+\left(T V \alpha_{1 L} / \beta_{1 L}\right) \\
& \times\left(\partial \ln V / \partial L_{1}\right)_{T, P}^{\mathrm{l}}-T V \alpha_{\mathrm{g}}\left(\partial \sigma_{\mathrm{g}} / \partial L_{1}\right) / 3
\end{aligned}
$$

Replacing $\left(\partial \ln V / \partial L_{1}\right)_{T, P}^{1}$ in eq 146 by $\left(\partial \Delta_{\mathrm{g}} \ln V /\right.$ $\left.\partial L_{1}\right)$ with eq 144 , one obtains an alternative form

$$
\begin{aligned}
& \partial \Delta_{\mathrm{g}} H / \partial L_{1} \\
&= f_{\mathrm{e}}+\left(\Delta C_{p}-T V \Delta \alpha \alpha_{1 L} / \beta_{1 L}\right)\left(\partial T / \partial L_{1}\right)_{\mathrm{g}} \\
&+\left(T V \alpha_{1 L} / \beta_{1 L}\right)\left(\partial \Delta_{\mathrm{g}} \ln V / \partial L_{1}\right) \\
&+T V\left(\beta_{\mathrm{g}} \alpha_{1 L} / \beta_{1 L}-\alpha_{\mathrm{g}}\right)\left(\partial \sigma_{\mathrm{g}} / \partial L_{1}\right) / 3
\end{aligned}
$$

Criteria for the Glass Transition and Thermodynamic Equations

The three criteria for the glass transition are here considered as in a previous paper ${ }^{1}$ :

(1) iso-hole fraction or iso-free volume (iso- $V_{\mathrm{f}}$ )

$$
(\mathrm{d} \tilde{V})_{\mathrm{g}}=0
$$

(2) iso-configurational entropy (iso- $S^{\mathrm{c}}$ )

$$
\left(\mathrm{d} S^{\mathrm{c}}\right)_{\mathrm{g}}=0
$$

(3) iso-configurational energy (iso- $U^{\mathrm{c}}$ )

$$
\left(\mathrm{d} U^{\mathrm{c}}\right)_{\mathrm{g}}=0
$$

The combination of eq 137, 131, and 136 with eq 148,149 , and 150 give the expressions for $\left(\partial T / \partial L_{1}\right)_{\mathrm{g}}$ for each criterion:

For iso- $V_{\mathrm{f}}$;

$$
\left(\partial T / \partial L_{1}\right)_{\mathrm{g}}=-(\Delta \beta / \Delta \alpha)\left(\partial \ln V / \partial L_{1}\right)_{T, P}^{1} / \beta_{1 L}
$$

For iso- $S^{c}$;

$$
\begin{aligned}
\left(\partial T / \partial L_{1}\right)_{\mathrm{g}}= & \left\{T(\partial f / \partial T)_{P, L}^{1}\right. \\
& \left.+T V \alpha_{\mathrm{g}}\left(\partial \ln V / \partial L_{1}\right)_{T, P}^{1}\right\} / \Delta C_{p} \\
= & \left\{f_{s}-T \Delta \alpha\left(\partial V / \partial L_{1}\right)_{T, P}^{1} / \beta_{1 L}\right\} / \Delta C_{p}
\end{aligned}
$$

For iso- $U^{\mathrm{c}}$;

$$
\begin{aligned}
\left(\partial T / \partial L_{1}\right)_{\mathrm{g}}= & \left\{f-T(\partial f / \partial T)_{p, L_{1}}^{1}\right. \\
& \left.+T V \alpha_{\mathrm{g}}\left(\partial \ln V / \partial L_{1}\right)_{T, P}^{1}\right\} / \Delta C_{p} \\
=\{ & \left.-f_{\mathrm{e}}-T \Delta \alpha\left(\partial V / \partial L_{1}\right)_{T, P}^{1} / \beta_{1 L}\right\} / \Delta C_{p}
\end{aligned}
$$

With these formulas of $\left(\partial T / \partial L_{1}\right)_{\mathrm{g}}$, the expressions of specific volume and enthalpy of the stretched glass for each criterion may be obtained from eq 144 and 146 or 147.

\section{Application of Statistical Theory of Rubber Elas- ticity}

One can apply the statistical theory of rubber elasticity to the present theory without any inconsistency. The results of the statistical theory required here are, with the use of Flory's expression, ${ }^{3}$

$$
\begin{aligned}
& f=G\left(V_{i} / V_{0}\right)^{2 / 3} L_{i}^{-1}\left(\lambda-\lambda^{-2}\right) \\
& f_{\mathrm{e}} / f=1-f_{s} \mid f=\mathrm{d} \ln \left\langle r^{2}\right\rangle_{0} / \mathrm{d} \ln T \\
& \left(\partial \ln V / \partial L_{1}\right)_{T, P}=\beta_{1 L} G V_{i}^{-1 / 3} V_{0}^{-2 / 3} \lambda^{-2} L_{i}^{-1}
\end{aligned}
$$

where $G=\nu k T$ ( $\nu$, number of effective network chains), $V_{i}$ and $L_{i}$ are the volume and length $L_{1}$ in the unstretched liquid state, i.e., at $\varepsilon=\varepsilon^{*} ; V_{0}$ is the volume when $\varepsilon=\varepsilon_{0}$ or $\boldsymbol{\gamma}=\mathbf{E}$ ( $\mathbf{E}$, unit tensor); $\left\langle r^{2}\right\rangle_{0}$ is unperturbed mean square end-to-end distance of a network chain corresponding to $\varepsilon_{0}{ }^{2}$; and $\lambda$ is $L_{1} / L_{i}$ equal to $\lambda_{1}$ defined in eq 28 and 29 under the condition of eq 94 . The shear modulus $G^{\prime}$ is then $G^{\prime}=G\left(V_{i} / V_{0}\right)^{2 / 3} / V$. Substituting eq 154 -156 to eq 144 and 146 , we obtain, noticing $\sigma=f L_{1} / V$,

$$
\begin{aligned}
\Delta_{\mathrm{g}} \ln V= & \Delta \alpha \Delta T_{\mathrm{g}}+G^{\prime}\left\{\beta_{1 L}\left(1-\lambda^{-1}\right)-\beta_{\mathrm{g}}\left(\lambda^{2}-\lambda^{-1}\right) / 3\right\} \\
\Delta_{\mathrm{g}} H= & G^{\prime} V\left(\mathrm{~d} \ln \left\langle r^{2}\right\rangle_{0} / \mathrm{d} \ln T\right) \\
& \times\left(\lambda^{2} / 2+\lambda^{-1}-3 / 2\right)+T V \alpha_{1 L} G^{\prime}\left(1-\lambda^{-1}\right) \\
& -T V \alpha_{\mathrm{g}} G^{\prime}\left(\lambda^{2}-\lambda^{-1}\right) / 3+\Delta C_{p} \Delta T_{\mathrm{g}}
\end{aligned}
$$


On the other hand, from eq $151-153$ with eq 154-156, one has the expressions for the change in glass transition temperature for each criterion:

For iso- $V_{\mathrm{f}}$;

$$
\Delta T_{\mathrm{g}}=-(\Delta \beta / \Delta \alpha) G^{\prime}\left(1-\lambda^{-1}\right)
$$

For iso- $S^{\mathrm{c}}$;

$$
\begin{aligned}
\Delta T_{\mathrm{g}}= & \left(G^{\prime} V / \Delta C_{p}\right)\left(1-\mathrm{d} \ln \left\langle r^{2}\right\rangle_{0} / \mathrm{d} \ln T\right) \\
& \times\left(\lambda^{2} / 2+\lambda^{-1}-3 / 2\right)-\left(G^{\prime} T V \Delta \alpha / \Delta C_{p}\right)\left(1-\lambda^{-1}\right)
\end{aligned}
$$

For iso- $U^{\mathrm{c}}$

$$
\begin{aligned}
\Delta T_{\mathrm{g}}= & -\left(G^{\prime} V / \Delta C_{p}\right)\left(\mathrm{d} \ln \left\langle r^{2}\right\rangle_{0} / \mathrm{d} \ln T\right) \\
& \times\left(\lambda^{2} / 2+\lambda^{-1}-3 / 2\right)-\left(G^{\prime} T V \Delta \alpha / \Delta C_{p}\right)\left(1-\lambda^{-1}\right)
\end{aligned}
$$

Substituting these formulas of $\Delta T_{\mathrm{g}}$ into eq 157 and 158 , one can derive the expressions of $\Delta_{\mathrm{g}} \ln V$ and $\Delta_{\mathrm{g}} H$ of the stretched glass for each criterion.

\section{DISCUSSION}

\section{Criteria for Glass Transition}

With the aid of eq $151-153$ or $159-161$, the three criteria for the glass transition may be checked by the measurements of the change in $T_{\mathrm{g}}$ with stretching, $\partial T_{\mathrm{g}} / \partial L_{1}\left[=\left(\partial T / \partial L_{1}\right)_{\mathrm{g}}\right]$. According to the iso-free volume assumption, i.e., eq $151, T_{\mathrm{g}}$ decreases with stretching since the number of holes, accordingly the free volume, increases with elongation because the volume dilatation is induced by stretching, i.e., $(\partial \ln V /$ $\left.\partial L_{1}\right)_{T, P}^{1}>0$. On the other hand, if the iso- $S^{\mathrm{c}}$ assumption is valid, $T_{\mathrm{g}}$ should increase by stretching as seen from eq 152 because $(\partial f / \partial T)_{P}{ }^{1}$ may be positive in network polymers. In the third case expressed by eq 153 , the change in $T_{\mathrm{g}}$ may be small, since $f-T(\partial f / \partial T)_{P, L_{1}}^{1}$ may be smaller than $f$ and the volume dilatation is also small. The term $f_{s}$ (or $-f_{\mathrm{e}}$ ) in eq $152^{\prime}$ (or $153^{\prime}$ ) indicates the contribution of the decrease in $S^{\mathrm{c}}$ (or $U^{\mathrm{c}}$ ) arising from the decrease in conformational entropy (or energy) of network chains with stretching, and the second term in the braces \{\} in eq $152^{\prime}$ (or $153^{\prime}$ ) indicates the contribution of the increase in $S^{\mathrm{c}}$ (or $U^{\mathrm{c}}$ ) arising from the increase in the free volume, in other words the number of holes, with stretching.

It has been suggested from the free volume theory that the relaxation time for microbrownian motion reduces with stretching. This leads to the prediction that $T_{\mathrm{g}}$ decreases by stretching: The fractional free volume $f$ is expressed as a function of temperature and strain $\varepsilon$, according to Ferry and Stratton, ${ }^{4}$ by

$$
f=f_{\mathrm{g}_{0}}+\alpha_{\mathrm{f}}\left(T-T_{\mathrm{g} 0}\right)+\left(\beta_{\mathrm{f}} / \beta\right)(1-2 \mu) \varepsilon
$$

where $T_{\mathrm{g} 0}$ is $T_{\mathrm{g}}$ at $\varepsilon=0, f_{\mathrm{g} 0}$ is $f$ at $T=T_{\mathrm{g}}$ and $\varepsilon=0$, and $\alpha_{\mathrm{f}}$ and $\beta_{\mathrm{f}}$ are the thermal expansion coefficient and compressibility of free volume respectively. If the free volume at $T_{\mathrm{g}}$ is kept constant, one has from eq 162

$$
T_{\mathrm{g}}-T_{\mathrm{g} 0}=-\left(\beta_{\mathrm{f}} / \beta\right)(1-2 \mu) \varepsilon / \alpha_{\mathrm{f}}
$$

If $\beta_{\mathrm{f}}=\Delta \beta$ and $\alpha_{\mathrm{f}}=\Delta \alpha$ are assumed, eq 163 is identical to eq 151 , when strain is small, since $\left(\partial \ln V / \partial \ln L_{1}\right)_{T, P}^{1}=1-2 \mu_{1}$, and $\left(L_{1}-L_{10}\right) / L_{1}=\varepsilon$.

On the other hand, Gee, et al., ${ }^{5}$ studied thermodynamically the $T_{\mathrm{g}}$ of stretched network polymers by introducing one ordering parameter $z$, and derived the expression for $\partial T_{\mathrm{g}} / \partial L_{1}$ based on the assumption that $z$ is constant at $T_{\mathrm{g}}$. The result obtained is

$$
\partial T_{\mathrm{g}} / \partial L_{1}=\left(T / \Delta C_{p}\right) \Delta(\partial f / \partial T)_{P, L_{1}}
$$

They made the approximation $T \Delta(\partial f / \partial T)_{P, L_{1}} \cong$ $T(\partial f / \partial T)_{P}^{1}, L_{1} \cong f$ in comparing with experiments.* Namely, they presented

$$
T_{\mathrm{g}}-T_{\mathrm{g} 0}=\frac{T_{\mathrm{g} 0}}{\Delta C_{p} T_{0}} \int_{L_{10}}^{L_{1}} f \mathrm{~d} L_{1}
$$

The term $\int_{L_{10}}^{L_{1}} f \mathrm{~d} L_{1}$ represents the work of stretching at $T_{0}$.

Equation 164 with $T \Delta(\partial f / \partial T)_{P, L_{1}}=T(\partial f / \partial T)_{P, L_{1}}^{1}$ is equivalent to eq 152 for iso- $S^{\mathrm{c}}$ in the present theory if the second term in eq 152 arising from the volume dilatation with stretching is ignored.

* The author does not agree with their approximation because no evidence is found for $(\partial f / \partial T)_{P}^{\mathrm{g}}, L_{1}$ $\ll(\partial f / \partial T)_{P, L_{1}}^{1}$. The fact that the term $(\partial f / \partial T)_{P, L_{1}}^{1}$ appears in the present theory instead of $\Delta(\partial f / \partial T)_{P, L_{1}}$ in eq 164 arises from the inequality between $\left(\mathrm{d} Z^{\mathrm{c}}\right)^{1}$ and $\Delta(\mathrm{d} Z)$ as expressed by eq 12 . For example, $\Delta\left(\partial a_{i} / \partial L_{1}\right)_{P, T} \neq 0$ and $\Delta\left(\partial l_{i}{ }^{+} / \partial L_{1}\right)_{P, T} \neq 0$. On the other hand, the change in the cell volume with temperature and pressure can be approximated to be the same both in the liquid and glassy states under low pressure if the free energy is expressed by eq $10 .^{1}$ This leads to eq 14 and 15 . 
DiMarzio $^{6}$ also evaluated the change in $T_{\mathrm{g}}$ with stretching based on the assumption of iso- $S^{\mathrm{c}}$ at $T_{\mathrm{g}}$ and the ideal rubber elasticity. The result obtained is identical to eq 160 when $f_{\mathrm{e}} / f=\mathrm{d} \ln$ $\left\langle r^{2}\right\rangle_{0} / \mathrm{d} \ln T=0$ and, if the second term indicating the effect of the volume expansion is disregarded: i.e.,

$$
\Delta T_{\mathrm{g}}=\left(G^{\prime} V / \Delta C_{p}\right)\left(\lambda^{2} / 2+\lambda^{-1}-3 / 2\right)
$$

The experimental facts show that the sign of $\left(\partial T_{\mathrm{g}} / \partial L_{1}\right)$ depends on the method of measurement of $T_{\mathrm{g}} \cdot{ }^{7}$ However, the glass transition temperature defined as the temperature at which the microbrownian motion is frozen-in, as discussed in previous papers, may correspond to $T_{\mathrm{g}}$ observed by the volume-temperature measurement under cooling at constant length. ${ }^{*}$ Such measurements were made by Gee, et al. ${ }^{5}$ According to their experiments, $T_{\mathrm{g}}$ increases with stretching approximately by the amount predicted from their equation, eq 165 . With the aid of their data, DiMarzio concluded from his theory, eq 166, that the iso- $S^{\mathrm{c}}$ assumption for $T_{\mathrm{g}}$ is valid. The result of the present theory also suggests that the iso- $S^{\mathrm{c}}$, not the iso- $V_{\mathrm{f}}$ and the iso- $U^{\mathrm{c}}$, is a valid criterion for $T_{\mathrm{g}}$, because the second term of eq $152^{\prime}$ and $f_{\mathrm{e}}$ may be small compared with $f$. The measurement of $T_{\mathrm{g}}$ under elevated pressure cannot distinguish between the two criteria, the iso- $S^{\mathrm{c}}$ and the iso- $U^{\mathrm{c}}$, as described in a preceding paper. ${ }^{1}$ The examination of $\left(\partial T_{\mathrm{g}} / \partial L_{1}\right)$, however, makes it possible.

In order to test the criteria for $T_{\mathrm{g}}$ in more detail, the $T_{\mathrm{g}}-L_{1}$ relation (from $T-V$ curve under constant $\left.L_{1}\right), \alpha, \beta, C_{p}$, and $f$ for a styrenebutadiene copolymer (butadiene content $=10 \mathrm{wt} \%$ ) were measured and compared with eq $159-161 .{ }^{8}$ As the result, it was found that the experiment supports the iso- $S^{\mathrm{c}}$ assumption for $T_{\mathrm{g}}$.

Inserting eq $151-153$ into eq 144 and 146, one has the expressions of $\Delta_{\mathrm{g}} \ln V$ and $\Delta_{\mathrm{g}} H$ for each criterion, by which one may also test the criteria with the measurements of specific volume and

* The glass transition temperature should depend on the cooling rate. For the sake of testing the criteria for $T_{\mathrm{g}}$, therefore, data using the same cooling rate must be used. $T_{\mathrm{g}}$ in the expressions of $\Delta_{\mathrm{g}} H$ and $\Delta_{\mathrm{g}} \ln V$ is not necessarily that of the same cooling rate, however, and implies only the glass-forming temperature. enthalpy of a stretched glass.

\section{Enthalpy of Streched Glass}

According to eq 147, the enthalpy of stretched glass depends not only on the conformational energy of stretched network chains expressed by $\int_{L_{10}}^{L_{1}} f_{\mathrm{e}} \mathrm{d} L_{1}$, but also on the freezing temperature, $T_{\mathrm{g}}$, and the specific volume of the glass. Stölting and Müller ${ }^{9}$ abtained the enthalpy difference $\Delta_{\mathrm{g}} \boldsymbol{H}$ of streched polystyrene glass by measurement of the heat of solution, and concluded that $\Delta_{\mathrm{g}} H$ for the glass stretched above $124^{\circ} \mathrm{C}$ is explained by the change in the energy arising from the variation in chain conformation with stretching. The author, however, believes that in general $\Delta_{\mathrm{g}} H$ is controlled not only by the change in the conformation with stretching, but also by the glassforming temperature and the specific volume of the glass, which are also the factors determining the energies associated with chain conformation and intersegmental interactions.

Finally it can be noted that eq 147 gives the method of evaluating the energetic elasticity $f_{\odot}$ from the measurements of $\Delta_{\mathrm{g}} H, \Delta_{\mathrm{g}} \ln V, \alpha_{\mathrm{g}}$, and $T_{\mathrm{g}}$.

Acknowledgment. The author wishes to thank Prof. T. Hata of the Tokyo Institute of Technology and the members of the Hata Laboratory for encouraging discussions and helpful advice.

\section{APPENDIX I}

From the equations of state, the quantities defined by eq $50-61$ can be expressed in terms of various $K$ defined by eq $39-42$ and $62-65$.

Differentiating eq 45 with temperature under constant pressure gives

$$
\begin{aligned}
& -V \alpha_{1 L} P \\
& =\left(\partial K_{2}{ }^{2} / \partial T\right)_{P, L_{1}}^{1}+\left(\partial K_{2}^{+} / \partial T\right)_{P, L_{1}}^{1} \\
& =\left(\partial K_{2}{ }^{2} / \partial T\right)_{P, L_{1}}^{1}+\tilde{K}(\partial \ln \tilde{V} / \partial T)_{P, L_{1}}^{1}-\left(\partial S_{2}^{\mathrm{c}} / \partial \ln \tilde{V}\right) \\
& =\left(\partial K_{2}{ }^{2} / \partial T\right)_{P, L_{1}}^{1}+\tilde{K}\left(\alpha_{1 L}-\alpha_{1 L}^{+}\right)-\left(\partial S_{2}{ }^{\mathrm{c}} / \partial \ln \tilde{V}\right)
\end{aligned}
$$

From eq 33 and 37 , one has

$$
\begin{gathered}
\sum_{k=1}^{3} K_{2 k}^{+}\left(\partial \ln l_{k}^{+} / \partial T\right)_{P, L_{1}}^{1}+\left(\partial K_{2}^{+} / \partial T\right)_{l_{1,2,3}^{+}} \\
=\tilde{K}(\partial \ln \tilde{V} / \partial T)_{P, L_{1}}^{1}-\left(\partial S_{2}{ }^{\mathrm{c}} / \partial \ln \tilde{V}\right)
\end{gathered}
$$

With the aid of eq 27 and 43, eq A-2 becomes 
Equation of State and Glass Transition of Stretched Network Polymers

$$
\begin{aligned}
& \alpha_{1 L}^{+} \sum_{k=1}^{3} K_{2 k}^{+} / 3+\left(\partial K_{2}^{+} / \partial T\right)_{l_{1,2,3}^{+}}^{+} \\
& \quad=\tilde{\widetilde{K}}\left(\alpha_{1 L}-\alpha_{1 L}^{+}\right)-\left(\partial S_{2}^{\mathrm{c}} / \partial \ln \tilde{V}\right)
\end{aligned}
$$

Then, from eq A-1 and A-3 one has eq 66 and 67;

$$
\begin{aligned}
\alpha_{1 L}^{+}= & 3\left\{\left(\partial K_{2}^{+} / \partial T\right)_{l_{1,2,3}^{+}}+\left(\partial K_{2}{ }^{\lambda} / \partial T\right)_{P, L_{1}}^{1}\right. \\
& \left.+P V \alpha_{1 L}\right\} / \sum_{k=1}^{3} K_{2 k}^{+}
\end{aligned}
$$

or

$$
\begin{aligned}
\alpha_{1 L}-\alpha_{1 L}^{+}= & \left\{\left(\partial S_{2}^{\mathrm{c}} / \partial \ln \tilde{V}\right)-\left(\partial K_{2}{ }^{2} / \partial T\right)_{P, L_{1}}^{1}\right. \\
& \left.-V P \alpha_{1 L}\right\} / \tilde{\tilde{K}}
\end{aligned}
$$

On the other hand, thermal expansion coefficient $\alpha_{\mathrm{g} L}$ of the glassy state is derived from eq 48 . Differentiating eq 48 with temperature under constant pressure and constant length, one has

$$
\begin{aligned}
- & V \alpha_{\mathrm{g} L} P \\
= & \left(\partial K_{2}^{a} / \partial T\right)_{P, L_{1}}^{g}+\left(\partial K_{2}^{+} / \partial T\right)_{P, L_{1}}^{g} \\
= & \sum_{k=1}^{3} K_{2 k}^{a}\left(\partial \ln a_{k} / \partial T\right)_{P, L_{1}}^{\mathrm{g}}+\left(\partial K_{2}^{a} / \partial T\right)_{a_{1,2,3}} \\
& \quad+\sum_{k=1}^{3} K_{2 k}^{+}\left(\partial \ln l_{k}^{+} / \partial T\right)_{P, L_{1}}^{g}+\left(\partial K_{2}^{+} / \partial T\right)_{l_{1,2,3}^{+}}
\end{aligned}
$$

Since $\left(\partial \ln a_{k} / \partial T\right)_{P, L_{1}}^{\mathrm{g}}=\left(\partial \ln l_{k}^{+} / \partial T\right)_{P, L_{1}}^{\mathrm{g}}=\alpha_{\mathrm{g} L} / 2$ for $k=2$ and 3 , and $\left(\partial \ln a_{1} / \partial T\right)_{P}^{g}, L_{1}=\left(\partial \ln l_{1}^{+} / \partial T\right)_{P}^{g}, L_{1}$ $=0$, eq A-4 reduces to eq 68 ;

$$
\begin{gathered}
\alpha_{\mathrm{g} L}=-\left\{\left(\partial K_{2}^{+} / \partial T\right)_{l_{1,2,3}^{+}}+\left(\partial K_{2}^{a} / \partial T\right)_{a_{1,2,3}}\right\} / \\
\left\{\sum_{k=2}^{3} K_{2 k}^{+} / 2+\sum_{k=2}^{3} K_{2 k}^{a} / 2+P V\right\}
\end{gathered}
$$

Similarly, eq 69 for $\alpha_{\mathrm{g}}$ is derived.

The isothermal compressibility $\beta_{1 L}$ at constant length in the liquid state is derived from eq 45 as $\beta_{1 L}^{-1} \equiv-(\partial P / \partial \ln V)_{T, L_{1}}^{1}=P+\left[\partial\left(K_{2}^{\lambda}+K_{2}^{+}\right) / \partial V\right]_{T, L_{1}}^{1}$

$=P+\sum_{k=1}^{3} K_{2 k}^{\lambda}\left(\partial \ln \lambda_{k} / \partial V\right)_{T, L_{1}}^{1}+\tilde{\widetilde{K}}(\partial \ln \tilde{V} / \partial V)_{T, L_{1}}^{1}$

$$
=P+P^{\lambda}+\tilde{K} V^{-1}\left\{1-\left(\partial \ln V^{+} / \partial \ln V\right)_{T, L_{1}}^{1}\right\}
$$$$
=\boldsymbol{P}+\boldsymbol{P}^{\lambda}+\tilde{\widetilde{K}}\left(1-\beta_{1 L}^{+} / \beta_{1 L}\right) / \boldsymbol{V}
$$

where $P^{\lambda}$ is defined by eq 78. Total differentials of $K_{2}^{+}$and $\tilde{K}$ are written, from the definition of eq 39 and 40 with the aid of eq 33 , as

$$
\begin{aligned}
& \mathrm{d} K_{2}^{+}=\sum_{k=1}^{3} K_{2 k} \mathrm{~d} \ln l_{k}^{+}+\left(\partial S_{2}^{+} / \partial \ln l_{2}^{+}\right)_{T} \mathrm{~d} T \\
& \mathrm{~d} \tilde{K}=\tilde{K} \mathrm{~d} \ln \tilde{V}+\left(\partial S_{2}{ }^{\mathrm{c}} / \partial \ln \tilde{V}\right) \mathrm{d} T
\end{aligned}
$$

Then from eq 45, A-7 and A-8, one has

$$
\sum_{k=1}^{3} K_{2 k}^{+}\left(\partial \ln l_{k}^{+} / \partial \ln P\right)_{T, L_{1}}^{1}=\tilde{K}(\partial \ln \tilde{V} / \partial \ln P)_{T, L_{1}}^{1}
$$

Since eq 43 holds in the liquid state, eq A-9 reduces to

$$
\beta_{1 L}^{+} \sum_{k=1}^{3} K_{2 k}^{+} / 3=\tilde{\widetilde{K}}\left(\beta_{1 L}-\beta_{1 L}^{+}\right)
$$

Accordingly, eq A-6 and A-10 yield eq 70 and 71:

$$
\begin{aligned}
& \beta_{1 L}^{+}=3 V\left\{1-\left(\boldsymbol{P}+\boldsymbol{P}^{\lambda}\right) \beta_{1 L}\right\} / \sum_{k=1}^{3} K_{2 k}^{+} \\
& \beta_{1 L}-\beta_{1 L}^{+}=V\left\{1-\left(\boldsymbol{P}+\boldsymbol{P}^{\lambda}\right) \beta_{1 L}\right\} / \widetilde{\boldsymbol{K}}
\end{aligned}
$$

On the other hand $\beta_{\mathrm{g} L}$ is expressed from eq 48 as

$$
\begin{aligned}
\beta_{\mathrm{g} L}^{-1}= & P+\sum_{k=1}^{3} K_{2 k}^{a}\left(\partial \ln a_{k} / \partial V\right)_{T, L_{1}}^{\mathrm{g}} \\
& +\sum_{k=1}^{3} K_{2 k}^{+}\left(\partial \ln l_{k}^{+} / \partial V\right)_{T}^{\mathrm{g}}, L_{1} \\
= & P+P^{a}+\sum_{k=2}^{3} K_{2 k}^{+} / 2 V
\end{aligned}
$$

where

$$
P^{a}=\sum_{k=1}^{3} K_{2 k}^{a}\left(\partial \ln a_{k} / \partial V\right)_{T, L_{1}}^{\mathrm{g}}=\sum_{k=2}^{3} K_{2 k}^{a} / 2 V
$$

and $\left(\mathrm{d} \ln l_{1}^{+}\right)_{L_{1}}^{g}=\left(\mathrm{d} \ln a_{1}\right)_{L_{1}}^{g}=0$ was used. Similarly, eq 70 for $\beta_{\mathrm{g}}$ is derived.

Next, $\left(\partial \ln V / \partial \ln L_{1}\right)_{T, P}^{1}=1-2 \mu_{1}$ is evaluated. From eq 45

$$
\begin{aligned}
& -V P\left(\partial \ln V / \partial L_{1}\right)_{T, P}^{1} \\
& =\left(\partial K_{2}{ }^{\lambda} \partial L_{1}\right)_{T, P}^{1}+\sum_{k=1}^{3} K_{2 k}^{+}\left(\partial \ln l_{k}{ }^{+} / \partial L_{1}\right)_{T, P}^{1}
\end{aligned}
$$

On the other hand, from eq 37 with eq A-7 and A-8, one has

$$
\sum_{k=1}^{3} K_{2 k}^{+}\left(\partial \ln l_{k}^{+} / \partial L_{1}\right)_{T, P}^{1}=\tilde{\widetilde{K}}\left(\partial \ln V / \partial L_{1}\right)_{T, P}^{1}
$$

Using the relation $\left(\partial \ln V / \partial L_{1}\right)_{T, P}^{1}=\left(\partial \ln \tilde{V} / \partial L_{1}\right)_{T, P}^{1}$ $+3\left(\partial \ln l_{k}^{+} / \partial L_{1}\right)_{T, P}$ derived from eq 23 and 43 , one obtains with eq A-12 and 77,

$$
\begin{aligned}
& \left(\partial \ln V / \partial \ln L_{1}\right)_{T, P}^{1} \\
& =-\frac{\left(\partial K_{2}^{\lambda} / \partial \ln L_{1}\right)_{T, P}^{1}\left(3 \tilde{\widetilde{K}}+\sum_{k=1}^{3} K_{2 k}^{+}\right)}{\tilde{\widetilde{K}} \sum_{k=1}^{3} K_{2 k}^{+}+P V\left(3 \tilde{K}+\sum_{k=1}^{3} K_{2 k}^{+}\right)}
\end{aligned}
$$

In the glassy state, eq 48 yields

$-V P\left(\partial \ln V / \partial \ln L_{1}\right)_{T, P}^{\mathrm{g}}$ 


$$
\begin{aligned}
= & \sum_{k=1}^{3} K_{2 k}^{a}\left(\partial \ln a_{k} / \partial \ln L_{1}\right)_{T, P}^{\mathrm{g}} \\
& +\sum_{k=1}^{3} K_{2 k}^{+}\left(\partial \ln l_{k}^{+} / \partial \ln L_{1}\right)_{T, P}^{\mathrm{g}}
\end{aligned}
$$

Since

$\left(\partial \ln a_{1} / \partial \ln L_{1}\right)_{T, P}^{\mathrm{g}}=\left(\partial \ln l_{1}^{+} / \partial \ln L_{1}\right)_{T, P}^{\mathrm{g}}=1$

and

$$
\begin{aligned}
\left(\partial \ln a_{k} / \partial \ln L_{1}\right)_{T, P}^{\mathrm{g}} & =\left(\partial \ln l_{k}^{+} / \partial \ln L_{1}\right)_{T, P}^{\mathrm{g}} \\
& =\left\{\left(\partial \ln V / \partial \ln L_{1}\right)_{T, P}^{\mathrm{g}}-1\right\} / 2
\end{aligned}
$$

for $k=2$ and 3 , then eq 75 can be derived as

$\left(\partial \ln V / \partial \ln L_{1}\right)_{T, P}^{\mathrm{g}}$

$$
\begin{aligned}
= & \left\{\sum_{k=2}^{3} K_{2 k}^{+} / 2+P^{a} V-\left(K_{21}^{a}+K_{21}^{+}\right)\right\} / \\
& \left\{\sum_{k=2}^{3} K_{2 k}^{+} / 2+P^{a} V+P V\right\} \\
= & 1-\left(K_{21}^{a}+K_{21}^{+}+P V\right) /\left(\sum_{k=2}^{3} K_{2 k}^{+} / 2+P^{a} V+P V\right)
\end{aligned}
$$

Young's modulus $E_{\mathrm{g}}=\left(\partial \sigma / \partial \ln L_{1}\right)_{T, P}^{\mathrm{g}, P}$ in the glassy state is obtained by differentiating eq 44 with the length $L_{1}$ under $T, P=$ constant:

$$
\begin{aligned}
& (\sigma-P)\left(\partial V / \partial \ln L_{1}\right)_{T}^{\mathrm{g}}, P+V E_{\mathrm{g}} \\
& =\sum_{k=1}^{3} K_{1 k}^{a}\left(\partial \ln a_{k} / \partial \ln L_{1}\right)_{T, P}^{\mathrm{g}} \\
& \quad+\sum_{k=1}^{3} K_{1 k}^{+}\left(\partial \ln l_{k}^{+} / \partial \ln L_{1}\right)_{T, P}^{\mathrm{g}}
\end{aligned}
$$

With the use of eq A-14 and A-15, eq A-17 reduces to eq 76 ;

$$
E_{\mathrm{g}}=\left\{K_{11}^{a}+K_{11}^{+}-2\left(K_{12}^{a}+K_{12}^{+}\right) \mu_{\mathrm{g}}\right\} / V-\left(1-2 \mu_{\mathrm{g}}\right)(\sigma-P)
$$

\section{APPENDIX II}

Since in the liquid state the total differential of $\ln V$ is written as

$$
\begin{aligned}
\mathrm{d} \ln V= & \left(\partial \ln V / \partial L_{1}\right)_{T, P}^{1} \mathrm{~d} L_{1}+(\partial \ln V / \partial T)_{P, L_{1}}^{1} \mathrm{~d} T \\
& +(\partial \ln V / \partial P)_{T, L_{1}}^{1} \mathrm{~d} P
\end{aligned}
$$

one has

$$
\begin{aligned}
\alpha_{1} \equiv & (\partial \ln V / \partial T)_{P, \sigma=0}^{1} \\
= & \left.\left(\partial \ln V / \partial L_{1}\right)_{T, P}^{1}\right|_{\sigma=0}\left(\partial L_{1} / \partial T\right)_{P, \sigma=0}^{1} \\
& +\left.(\partial \ln V / \partial T)_{P, L_{1}}^{1}\right|_{\sigma=0}
\end{aligned}
$$

The symbol $\left.\right|_{\sigma=0}$ indicates the value at $\sigma=0$. From the well known relation $1-2 \mu=E \beta / 3$ for isotropic substances, $\left.\left(\partial \ln V / \partial L_{1}\right)_{T, P}^{1}\right|_{\sigma=0}$ is $E \beta / 3$, and $3(\partial \ln$ $\left.L_{1} / \partial T\right)\left._{P}^{1}\right|_{\sigma=0}=\alpha_{1}$, because of isotropy. One has then from eq A-19

$$
\alpha_{1}\left(1+E_{1} \beta_{1} / 9\right)=\left.\alpha_{1 L}\right|_{\sigma=0}
$$

Under the condition of eq $95, E_{1} \beta_{1} \ll 1$. Accordingly, it follows from eq A-20 that

$$
\alpha_{1}=\left.\alpha_{1 L}\right|_{\sigma=0}
$$

On the other hand, one can expand $\alpha_{1 L}$ at $\sigma=0$ as

$$
\begin{aligned}
\alpha_{1 L}= & \left.\alpha_{1 L}\right|_{\sigma=0}+\left.\left(\partial^{2} \ln V / \partial \ln L_{1} \partial T\right)_{P}^{1}\right|_{\sigma=0} \\
& \times \ln \left(L_{1} / L_{10}\right)+\cdots
\end{aligned}
$$

where $L_{10}$ is the length $L_{1}$ at $\sigma=0$. Since $(\partial \ln V /$ $\left.\partial \ln L_{1}\right)\left._{P, T}^{1}\right|_{\sigma=0}=E_{1} \beta_{1} / 3 \quad$ and $\quad\left[\partial\left(E_{1} \beta_{1}\right) / \partial T\right]_{P, L_{1}}=$ $\mathrm{O}\left(E_{1} \beta_{1} / T\right)$, then from eq A-22

$$
\alpha_{1 L}=\left.\alpha_{1 L}\right|_{\sigma=0}+\mathbf{O}\left(\beta_{1} \sigma / T\right)=\left.\alpha_{1 L}\right|_{\sigma=0}\left\{1+\mathbf{O}\left(\sigma / \boldsymbol{P}_{\mathrm{i}}\right)\right\}
$$

Under the condition of eq 96 (i.e., $\sigma \ll P_{\mathrm{i}}$ ) with eq $\mathrm{A}-21$, eq $\mathrm{A}-23$ reduces to

$$
\alpha_{1 L}=\left.\alpha_{1 L}\right|_{\sigma=0}=\alpha_{1}
$$

Similarly to eq A-21, one has under eq 95

$$
\beta_{1} \equiv-(\partial \ln V / \partial P)_{T, \sigma=0}^{1}=\left.\beta_{1 L}\right|_{\sigma=0}
$$

Corresponding to eq A-22, one has for $\beta_{1 L}$

$$
\begin{aligned}
\beta_{1 L}= & \left.\beta_{1 L}\right|_{g=0}+\left.\left(\partial^{2} \ln V / \partial \ln L_{1} \partial P\right)_{T}^{1}\right|_{o=0} \\
& \times \ln \left(L_{1} / L_{10}\right)+\cdots
\end{aligned}
$$

As seen from eq 100 and 101 with eq 121 and $122, \beta_{1 L}$ depends on volume and temperature alone in the present approximations. Hence it follows that

$$
\begin{aligned}
& \left.\left(\partial^{2} \ln V / \partial \ln L_{1} \partial P\right)_{T}^{1}\right|_{\sigma=0} \\
& \quad=-\left.\left(\partial \beta_{1 L} / \partial \ln V\right)_{T, L_{1}}^{1}\left(\partial \ln V / \partial \ln L_{1}\right)_{P, T}\right|_{\sigma=0}
\end{aligned}
$$

Then, from eq A-25, A-26 and A-27, one has

$$
\begin{aligned}
\beta_{1 L}= & \left.\beta_{1 L}\right|_{\sigma=0}+\left.\left(\partial \ln \beta_{1} / \partial P\right)_{T,}^{1}\right|_{\sigma=0} \\
& \times\left(E_{1} \beta_{1} / 3\right) \ln \left(L_{1} / L_{10}\right)+\cdots \\
= & \left.\beta_{1 L}\right|_{\sigma=0}+\beta_{1} \mathrm{O}\left[\sigma\left(\partial \ln \beta_{1} / \partial P\right)_{T, \sigma=0}\right]
\end{aligned}
$$

For liquids one can except $\sigma\left(\partial \ln \beta_{1} / \partial P\right)_{T, \sigma=0}=$ $\mathrm{O}(10 \sigma \beta) \ll 1$, as estimated from Tait's equation, ${ }^{10}$. $1-\left(V / V_{0}\right)=C \ln (1+P / B)$, which reproduces the experimental $V-P$ relation under constant $T$ very well, because the relation $(\partial \ln \beta / \partial P)_{T}=\left(V / V_{0}\right)$ $\times\left\{\left(V / V_{0} C\right)+1\right\} \beta \cong(1 / C+1) \beta \approx \beta / C$ is obtained from Tait's equation where $V_{0}$ is $V$ at $P=0, C$. 
is a constant, about 0.1 , and $B$ is a function of $T$ alone. Accordingly, one has finally from eq A-25 and A-28

$$
\beta_{1 L}=\left.\beta_{1 L}\right|_{\sigma=0}=\beta_{1}
$$

As for heat capacity,

$$
\begin{aligned}
C_{p 1} & \left.\equiv T(\partial S / \partial T)_{P}^{1}\right|_{\sigma=0} \\
& =T\left(\partial S / \partial \ln L_{1}\right)_{T, P}^{1}\left(\partial \ln L_{1} / \partial T\right)_{P,\left.\right|_{\sigma=0} ^{1}}+\left.C_{p 1 L}\right|_{\sigma=0}
\end{aligned}
$$

Since one may expect $T\left(\partial S / \partial \ln L_{1}\right)_{T, P}^{1}=\mathrm{O}\left(f L_{1}\right)$ and $C_{p 1}=\mathbf{O}\left(T V \alpha_{1}{ }^{2} / \beta_{1}\right)$, the first term of eq A-30 is estimated as

$$
\begin{aligned}
& T\left(\partial S / \partial \ln L_{1}\right)_{T, P}\left(\partial \ln L_{1} / \partial T\right)_{P, \sigma=0} \\
& \quad=\mathbf{O}\left(f L_{1}\right) \alpha_{1} / 3 \\
& \quad=C_{p 1} \mathrm{O}\left(f L_{1} \alpha_{1} \beta_{1} / T V \alpha_{1}{ }^{2}\right) \\
& \quad=C_{p 1} \mathrm{O}\left(\sigma / P_{\mathrm{i}}\right)
\end{aligned}
$$

Then, under the condition of eq 96, it follows from eq A-30 and A-31 that

$$
C_{p 1}=\left.C_{p 1 L}\right|_{\sigma=0}
$$

Just the same as eq A-22,

$C_{p 1 L}=\left.C_{p 1 L}\right|_{\sigma=0}+T\left(\partial^{2} S / \partial \ln L_{1} \partial T\right)_{P}^{1} \ln \left(L_{1} / L_{10}\right)+\cdots$

Since one may put $T\left(\partial S / \partial \ln L_{1}\right)_{T, F}^{1}=\mathrm{O}\left(f L_{1}\right)$ again and $(\partial f / \partial T)_{P, L_{1}}=\mathbf{O}(f / T)$, the second term of eq A-33 is evaluated as

$$
\begin{aligned}
\left.T\left(\partial S / \partial \ln L_{1} \partial T\right)_{P}^{1}\right|_{\sigma=0} & =\mathrm{O}\left(f L_{1} / T\right) \ln \left(L_{1} / L_{10}\right) \\
& =\mathrm{O}\left[f\left(L_{1}-L_{10}\right) / T\right]
\end{aligned}
$$

Then, with the same consideration as in the evaluation of eq A-31, one has, from eq A-32 A-34 under the condition of $\sigma /\left(P_{\mathrm{i}} / \alpha T\right) \ll 1$,

$$
C_{p 1 L}=\left.C_{p 1 L}\right|_{\sigma=0}=C_{p 1}
$$

With the same arguments as the above, one may show that the dependence of $\alpha_{1 L}^{+}, \beta_{1 L}^{+}$, and $C_{p 1 L}^{+}$ on the length $L_{1}$ can be ignored under the conditions of eq 95 and 96 .

As far as the form of free energy of eq 33, where $F_{2}$ representing the liquidlike properties is not a function of $\lambda$ and $\boldsymbol{a}$ but a function of volume and temperature alone in the liquid state, the dependence of $\alpha_{1 L}, \beta_{1 L}$, and $C_{p 1 L}$ on the length can be ignored from the fact that the Young's modulus is sufficiently lower than the bulk modulus and the internal pressure in the liquid state.

\section{REFERENCES}

1. T. Nose, Polymer J., 2, 445 (1971).

2. J. Meixner, Kolloid-Z., 134, 3 (1953); R. O. Davies and G. O. Jones, Proc. Roy. Soc., A217, 26 (1953), Advances in Physics, 2, 370 (1953).

3. P. J. Flory, Trans. Faraday Soc., 67, 829 (1961).

4. J. D. Ferry and R. A. Stratton, Kolloid-Z., 171, 107 (1960).

5. G. Gee, P. N. Hartley, T. B. M. Herbert, and H. A. Lanceley, Polymer, 1, 365 (1960).

6. E. A. DiMarzio, J. Res. Nat. Bur. Std., 68A, 611 (1964).

7. T. Nose, J. Soc. Rubber Ind., Japan, 44, 961 (1971).

8) A. Komatsu and T. Nose, Rept. Progr. Polym. Phys. Japan, 14, 309 (1971).

9) J. Stölting and F. H. Müller, Kolloid-Z. Polym., 238, 459 (1970); ibid., 240, 792 (1970).

10. W. G. Cutler, H. McMicklo, W. Weff, and R. W. Sciessler, J. Chem. Phys., 29, 272 (1958); V.S. Nanda and R. Simha, J. Chem. Phys., 41, 1884, 3870 (1964); J. Becht, K. H. Hellwege, and W. Knappe, Kolloid-Z., 216-217, 150 (1967); H. Breuer and G. Rehage, ibid., 216-217, 159 (1967). 